\title{
ON POLARIZED MANIFOLDS OF SECTIONAL GENUS THREE
}

\author{
HIRONOBU ISHIHARA \\ Department of Mathematics, Faculty of Science, Tokyo Institute of Technology
}

\section{Introduction.}

Let $L$ be an ample line bundle on a complex projective manifold $M$ of dimension $n \geq 2$. The sectional genus $g=g(M, L)$ of a polarized manifold $(M, L)$ is defined by the formula $2 g(M, L)-2=(K+(n-1) L) L^{n-1}$, where $K$ is the canonical bundle of $M$. For polarized manifolds over $\mathbb{C}$, it is known that $g$ takes non-negative integers ([F6;Corollary 1]).

In many papers the structure of $(M, L)$ with low $g$ has been studied: see [F6] for $g \leq 1$; [BeLP] for $g=n=2$; [F7] for $g=2$; [Ma] for $g=3$ and $n=2$. In this paper we study the case $g=3$ and $n \geq 3$. Under the additional condition that $L$ is spanned,the classification was partially known by [BiLL]. Here we study $(M, L)$ without this hypothesis.

This paper is organized as follows. In $\S 1$ we show that $(M, L)$ with $g=3$ and $n \geq 3$ is one of the following types.

(1) There is an effective divisor $E$ on $M$ such that $\left(E, L_{E}\right) \simeq\left(\mathbb{P}^{n-1}, \mathcal{O}(1)\right)$ and $[E]_{E}=\mathcal{O}(-1)$.

(2) There is a fibration $\Phi: M \rightarrow C$ over a smooth curve $C$ such that $\left(F, L_{F}\right) \simeq$ $\left(\mathbb{P}^{2}, \mathcal{O}(2)\right)$ for every fiber $F$ of $\Phi$.

(3) There is a fibration $\Phi: M \rightarrow C$ over a smooth curve $C$ such that every fiber $F$ of $\Phi$ is a hyperquadric in $\mathbb{P}^{n}$ and $L_{F}=\mathcal{O}(1)$.

(4) $(M, L)$ is a scroll over a smooth surface.

(5) $K+(n-2) L$ is nef.

(6) $(M, L)$ is a scroll over a smooth curve of genus three.

In $\S 2$ we study the case (4),in $\S 3$ we study the case $(3)$, in $\S 4$ we study the case $(2)$, and in $\S 5$ we study the cases (1) and (5). Although our results are far from being complete, they are very similar to those in case $g=2$.

The author would like to express his sincere thanks to Professor T. Fujita for kind encouragement and for many valuable comments during the preparation of this paper.

\section{Notation.}

Basically we use the customary notation in algebraic geometry as in [H2]. All varieties are defined over $\mathbb{C}$ and assumed to be complete. Vector bundles are often identified with locally free sheaves of their sections, and these words are used interchangeably. Line bundles are identified with linear equivalence classes of Cartier divisors, and their tensor products are denoted additively, while we use multiplicative notation for intersection products in Chow rings. The numerical equivalence 
of line bundles is denoted $\equiv$, while we use $=$ for linear equivalence. The linear equivalence class is denoted by [ ], and its corresponding invertible sheaf is denoted by $\mathcal{O}[]$.

Given a morphism $f: X \rightarrow Y$ and a line bundle $A$ on $Y$, we denote $f^{*} A$ by $A_{X}$,or sometimes by $A$ for short when there is no danger of confusion. The canonical bundle of a manifold $M$ is denoted by $K^{M}$, unlike the customary notation $K_{M}$. The $\mathcal{O}(1)$ 's of projective spaces $\mathbb{P}_{\alpha}, \mathbb{P}_{\beta}, \ldots$ will be denoted by $H_{\alpha}, H_{\beta}, \ldots$ Given a vector bundle $\mathcal{E}$ on $X$, we denote by $\mathbb{P}_{X}(\mathcal{E})($ or $\mathbb{P}(\mathcal{E})$ ) the associated projective space bundle,and denote by $H(\mathcal{E})$ the tautological line bundle on $\mathbb{P}(\mathcal{E})$ in the sense of [H2]. The pair $(\mathbb{P}(\mathcal{E}), H(\mathcal{E}))$ is called the scroll of $\mathcal{E}$.

\section{$\S 1$ Classification; first step.}

Throughout this paper a polarized manifold $(M, L)$ is a pair of a nonsingular projective variety $M$ over $\mathbb{C}$ and an ample line bundle $L$ on $M$. We consider the case with $n=\operatorname{dim} M \geq 3$ and denote by $K$ the canonical bundle of $M$. First we review known results about polarized manifolds.

(1.1)Theorem([F6;Theorem 1]). Let $(M, L)$ be a polarized manifold. Then $K+n L$ is nef unless $(M, L) \simeq\left(\mathbb{P}^{n}, \mathcal{O}(1)\right)$.

(1.2)Theorem([F6;Theorem 2]). Let $(M, L)$ be a polarized manifold with $n \geq 2$. Suppose that $K+n L$ is nef. Then $K+(n-1) L$ is nef unless either

(a) $M$ is a hyperquadric in $\mathbb{P}^{n+1}$ and $L=\mathcal{O}(1)$,

(b) $(M, L) \simeq\left(\mathbb{P}^{2}, \mathcal{O}(2)\right)$, or

(c) $(M, L)$ is a scroll over a smooth curve of genus $g(M, L)$.

We denote by $g=g(M, L)$ the sectional genus of $(M, L)$. In the above cases (a) and (b),we have $g=0$. Thus we obtain

(1.3)Corollary. For a polarized manifold $(M, L)$ with $g=3$ and $n \geq 2$, if $K+$ $(n-1) L$ is not nef, then $(M, L)$ is a scroll over a smooth curve of genus three.

When $K+(n-1) L$ is nef,we use the following theorem.

(1.4)Theorem([F6;Theorem 3]). Let $(M, L)$ be a polarized manifold with $n \geq 3$. Suppose that $K+(n-1) L$ is nef. Then $K+(n-2) L$ is nef except the following cases.

(a) There is an effective divisor $E$ on $M$ such that $\left(E, L_{E}\right) \simeq\left(\mathbb{P}^{n-1}, \mathcal{O}(1)\right)$ and $[E]_{E}=\mathcal{O}(-1)$.

(b0) $(M, L)$ is a Del Pezzo manifold (i.e. $K+(n-1) L=0),\left(\mathbb{P}^{3}, \mathcal{O}(2)\right),\left(\mathbb{P}^{3}, \mathcal{O}(3)\right)$, $\left(\mathbb{P}^{4}, \mathcal{O}(2)\right)$, or a hyperquadric in $\mathbb{P}^{4}$ with $L=\mathcal{O}(2)$.

(b1) There is a fibration $\Phi: M \rightarrow C$ over a smooth curve $C$ with one of the following properties:

(b1-V) $\left(F, L_{F}\right) \simeq\left(\mathbb{P}^{2}, \mathcal{O}(2)\right)$ for every fiber $F$ of $\Phi$;

(b1-Q) every fiber $F$ of $\Phi$ is a hyperquadric in $\mathbb{P}^{n}$ and $L_{F}=\mathcal{O}(1)$.

(b2) $(M, L)$ is a scroll over a smooth surface.

In the case of (b0),we have $g \neq 3$. Thus when $g=3$, Theorem (1.4) is rephrased as below.

(1.5)Theorem. For a polarized manifold $(M, L)$ with $g=3$ and $n \geq 3$, if $K+(n-$ 1) $L$ is nef,then $(M, L)$ is one of the following types.

(1) There is an effective divisor $E$ on $M$ such that $\left(E, L_{E}\right) \simeq\left(\mathbb{P}^{n-1}, \mathcal{O}(1)\right)$ and 
(2) There is a fibration $\Phi: M \rightarrow C$ over a smooth curve such that $\left(F, L_{F}\right) \simeq$ $\left(\mathbb{P}^{2}, \mathcal{O}(2)\right)$ for every fiber $F$ of $\Phi$.

(3) There is a fibration $\Phi: M \rightarrow C$ over a smooth curve such that every fiber $F$ of $\Phi$ is a hyperquadric in $\mathbb{P}^{n}$ and $L_{F}=\mathcal{O}(1)$.

(4) $(M, L)$ is a scroll over a smooth surface.

(5) $K+(n-2) L$ is nef.

We study the above cases in the following sections; in $§ 2$ we study the case (4), in $\S 3$ we study the case $(3)$, in $\S 4$ we study the case $(2)$, and in $\S 5$ we study the cases (1) and (5).

\section{$\S 2$ The case of a scroll over a surface.}

In this section we study the case (4) of the theorem (1.5), following the idea in $[\mathrm{F} 8 ; \S 2]$. From the definition of scrolls, we have $(M, L) \simeq\left(\mathbb{P}_{S}(\mathcal{E}), H(\mathcal{E})\right)$ for some ample vector bundle $\mathcal{E}$ on a smooth surface $S$.

(2.1) Since $\mathcal{E}$ is ample, $A:=\operatorname{det} \mathcal{E}$ is ample and $(S, A)$ is a polarized surface. A simple computation shows $g(S, A)=g(M, L)=3$, thus the classification is reduced to the classification of polarized surfaces with $g=3$.

(2.2) We first recall the definition of the minimalization of polarized surfaces (For details we refer to $[\mathrm{F} ; \S 14]$.). Let $(S, A)$ be a polarized surface. For a $(-1)$-curve $E$ on $S$,let $\pi: S \rightarrow S^{-}$be the contraction of $E$. Then $A+m E=\pi^{*} A^{-}$for an ample line bundle $A^{-}$on $S^{-}$and $m:=A E$ is called the weight of the contraction $\pi:(S, A) \rightarrow\left(S^{-}, A^{-}\right) . \pi$ is said to be admissible if $A Z \geq m$ for any $(-1)$-curve $Z$ on $S$. After a finite sequence of admissible contractions: $(S, A)=\left(S_{0}, A_{0}\right) \stackrel{\pi_{1}}{\longrightarrow}$ $\left(S_{1}, A_{1}\right) \stackrel{\pi_{2}}{\longrightarrow} \ldots \stackrel{\pi_{r}}{\longrightarrow}\left(S_{r}, A_{r}\right)=\left(S^{\prime}, A^{\prime}\right)$, we obtain that either $\left(S^{\prime}, A^{\prime}\right)$ is a $\mathbb{P}^{1}$ bundle over a curve or the canonical bundle $K^{\prime}$ of $S^{\prime}$ is nef. $\left(S^{\prime}, A^{\prime}\right)$ is called an admissible minimalization of $(S, A)$. We stop when $S^{\prime} \simeq \Sigma_{1}$ although there is a $(-1)$-curve on $S^{\prime}$. The weight sequence of this admissible minimalization is defined to be $\mathfrak{m}:=\left(m_{r}, \ldots, m_{1}\right)$, where $m_{j}(1 \leq j \leq r)$ is the weight of $\pi_{j} . \mathfrak{m}$ is known to be an invariant of $(S, A)$ and is independent of the choice of the minimalization process.

Polarized surfaces with $g=3$ are classified in [Ma].

(2.3)Theorem(cf. [Ma]). Let $(S, A)$ be a polarized surface. Taking an admissible minimalization of $(S, A):(S, A)=\left(S_{0}, A_{0}\right) \rightarrow\left(S_{1}, A_{1}\right) \rightarrow \cdots \rightarrow\left(S_{r}, A_{r}\right)=$ $\left(S^{\prime}, A^{\prime}\right)$, we denote by $\mathfrak{m}=\left(m_{r}, \ldots, m_{1}\right)$ its weight sequence. We put $\left(S^{\prime}, A^{\prime}\right)=$ $(S, A)$ when we need not take a minimalization. Assume that $g(S, A)=3$ and $A=\operatorname{det} \mathcal{E}$ for some ample vector bundle $\mathcal{E}$ with $\operatorname{rank} \mathcal{E} \geq 2$. Then $(S, A)$ is one of the following types.

(I) $K \equiv A$ and $A^{2}=2$.

(II) $S$ is a minimal surface of general type, $K^{2}=1, K A=2$, and $A^{2}=2$.

(III) $S$ is a minimal elliptic surface and $\left(A^{2}, K A\right)=(2,2)$ or $(3,1)$.

(IV) $K^{\prime} \equiv 0 . A^{2}, A^{\prime 2}$ and $\mathfrak{m}$ are as follows:

$$
\begin{array}{cccc} 
& A^{2} & A^{\prime 2} & \mathfrak{m}=\left(m_{r}, \ldots, m_{1}\right) \\
1) & 4 & & (2) \\
2) & 2 & 6 &
\end{array}
$$

(V) There is a vector bundle $\mathcal{F}$ of rank two on an elliptic curve $C$ such 
We put $e=c_{1}(\mathcal{F})$ and $y=\operatorname{deg} B$. Then $A^{2}, e, x, y$ and $\mathfrak{m}$ are as follows.

$\begin{array}{cccccc} & A^{2} & e & x & y & \mathfrak{m}=\left(m_{r}, \ldots, m_{1}\right) \\ 1) & 8 & 0,1 & 2 & 2-e & \\ 2) & 6 & 0 & 3 & 1 & \\ 3) & 5 & 1 & 5 & -2 & \\ 4) & 4 & 0,1 & 4 & 1-2 e & (2) \\ 5) & 3 & 0,1 & 6 & 1-3 e & (3) \\ 6) & 3 & 1 & 7 & -3 & (2) \\ 7) & 2 & 0 & 9 & 1 & (4) \\ 8) & 2 & 1 & 11 & -5 & (3) \\ 9) & 2 & 0 & 5 & 1 & (2,2)\end{array}$

Remark. Although $\mathcal{F}$ is normalized in $[\mathrm{Ma}]$, here we choose $\mathcal{F}$ satisfying $c_{1}(\mathcal{F})=$ 0 or 1 by tensoring some line bundle.

(VI) $(S, A) \simeq\left(\mathbb{P}^{2}, \mathcal{O}(4)\right)$.

(VII) $S^{\prime} \simeq \Sigma_{e}:=\mathbb{P}\left(\mathcal{O}_{\mathbb{P}^{1}} \oplus \mathcal{O}_{\mathbb{P}^{1}}(e)\right)$. We denote by $H$ the tautological bundle on $\Sigma_{e}$ and by $H_{\xi}$ the pullback of $\mathcal{O}_{\mathbb{P}^{1}}$ on $\Sigma_{e}$. Then we have $A^{\prime}=$ $x H+y H_{\xi}$, where $x$ and $y$ are integers and $E$ is the exceptional curve on $\Sigma_{e} . A^{2}, x, y, r$, and $\mathfrak{m}$ are as follows:
$A^{2}$
e $\quad x$
$\begin{array}{lll}x & y\end{array}$
$\mathfrak{m}=\left(m_{r}, \ldots, m_{1}\right)$
1) 16
$0,1,2 \quad 2 \quad 4-e \quad 0$
2) 4
$0,1 \quad 4 \quad 5-2 e \quad 9$
$(2, \ldots, 2)$
3) 3
$0,1 \quad 6 \quad 7-2 e \quad 9$
$(3, \ldots, 3)$

(VIII) There is an integer $j(0 \leq j \leq r)$ such that $\left(S_{j},-K_{j}\right)$ is a Del Pezzo surface, where $K_{j}$ is the canonical bundle of $S_{j}$, and $A_{j}=-a K_{j}$ for some integer $a . A^{2}, K_{j}^{2}$, a, and $\left(m_{j}, \ldots, m_{1}\right)$ are as follows:

$\begin{array}{ccccc} & A^{2} & K_{j}^{2} & a & \left(m_{j}, \ldots, m_{1}\right) \\ 1) & 8 & 2 & 2 & \\ 2) & 5 & 1 & 3 & (2) \\ 3) & 3 & 1 & 4 & (3,2) \\ 4) & 2 & 2 & 5 & (4,4,4) \\ 5) & 2 & 2 & 3 & (2,2,2,2) \\ 6) & 2 & 1 & 6 & (5,3)\end{array}$

Proof. The assertion easily follows from [Ma] and the next lemma.

(2.4)Lemma. Let $(S, A)$ be a polarized surface and $\mathcal{E}$ a vector bundle of rank $n-1$ on $S$ such that $(M, L) \simeq\left(\mathbb{P}_{S}(\mathcal{E}), H(\mathcal{E})\right)$ and $\operatorname{det} \mathcal{E}=A$. Then $A^{2}=L^{n}+c_{2}(\mathcal{E}) \geq 2$ and $A Z \geq \operatorname{rank} \mathcal{E} \geq 2$ for any rational curve $Z$ on $S$.

For a proof of this lemma,see $[\mathrm{F} 8 ;(2.2) \&(1.3)]$.

From now on,for some types of $(S, A)$ in the above list, we would like to classify ample vector bundles $\mathcal{E}$ such that $\operatorname{det} \mathcal{E}=A$.

(2.5) Suppose that $(S, A)$ is of the type $(2.3 ; \mathrm{I})$. From $(2.4)$ we have $L^{n}=c_{2}(\mathcal{E})=$ 1. Since $K \equiv A$ is ample, $S$ is a minimal surface of general type. By [Bo;Theorem 9], $p_{g}=h^{0}(S, K) \leq 3$ and we have the following possibilities: 
b) when $p_{g}=1, q \leq 1$ by e.g. [Be;Théorème X.4];

c) when $p_{g}=2, q=0$ by [Bo;Theorem 12];

d) when $p_{g}=3, q=0$ by [Bo;Theorem 10].

(2.6) Suppose that $(S, A)$ is of the type (2.3;II). From (2.4) we have $L^{n}=c_{2}(\mathcal{E})=$ 1. Since $K^{2}=1$,we have $p_{g} \leq 2$ by [Bo;Theorem 9] and $q=0$ by [Bo;Lemma 14]. Moreover,we can rule out the possibility that $p_{g}=2$. Assume that $p_{g}=2$.Then $\chi\left(\mathcal{O}_{S}\right)=3$, where $\chi$ is the Euler characteristic. From this,we obtain $\chi(A-K)=2$ by the Riemann-Roch theorem. Since $A(A-K)=0$, we have $h^{0}(A-K)=0$, thus $h^{2}(A-K)>0$ from $\chi(A-K)=2$. This means $h^{0}(2 K-A)>0$ by Serre duality. Since $K(2 K-A)=0$ and $A(2 K-A)=2$, any member of $|2 K-A|$ is one (-2)-curve. Hence we have $h^{0}(2 K-A) \leq 1$. This means $h^{2}(A-K) \leq 1$, thus $\chi(A-K) \leq 1$. This is a contradiction, hence $p_{g} \leq 1$.

(2.7) Suppose that $(S, A)$ is of the type $(2.3 ; \mathrm{V}-1)$. We treat this case similarly as in $[\mathrm{F} 8 ;(2.4) \&(2.5)]$. For every fiber $F$ of the bundle map $\rho: \mathbb{P}_{C}(\mathcal{F}) \rightarrow C$, we have $F \simeq \mathbb{P}^{1}$ and $A F=2$, hence rank $\mathcal{E}=2$ and $\mathcal{E}_{F} \simeq \mathcal{O}(1) \oplus \mathcal{O}(1)$. Then $\mathcal{G}:=\rho_{*}(\mathcal{E} \otimes[-H(\mathcal{F})])$ is a locally free sheaf of rank two on $C$ and $\rho^{*} \mathcal{G} \simeq \mathcal{E} \otimes[-H(\mathcal{F})]$. Moreover we have $M=\mathbb{P}_{S}(\mathcal{E}) \simeq S \times_{C} \mathbb{P}(\mathcal{G}), c_{1}(\mathcal{G})=\operatorname{deg} B=2-e, c_{2}(\mathcal{E})=2$, and $L^{3}=6$.

(2.7.1) When $e=0$, both $\mathcal{F}$ and $\mathcal{G}$ are semistable. In fact,for any quotient line bundle $Q$ of $\mathcal{F}$, denote by $Z$ the section of $\rho$ corresponding to $Q$. Then $c_{1}(Q) \geq 0$ since $0<A Z=\left(2 H(\mathcal{F})+B_{S}\right) Z=2 c_{1}(Q)+2$. Thus $\mathcal{F}$ is semistable. If $\mathcal{G}$ is not semistable,there exists a quotient line bundle $Q$ of $\mathcal{G}$ such that $2 c_{1}(Q)<c_{1}(\mathcal{G})$. Then $\rho^{*} Q \otimes H(\mathcal{F})$ is a quotient line bundle of $\mathcal{E}$. Hence $\rho^{*} Q \otimes H(\mathcal{F})$ is ample and $0<c_{1}^{2}\left(\rho^{*} Q \otimes H(\mathcal{F})\right)=2 c_{1}(Q)<c_{1}(\mathcal{G})=2$. This is a contradiction, thus $\mathcal{G}$ is semistable. When $e=1$, the semistability of $\mathcal{F}$ and $\mathcal{G}$ is uncertain.

(2.7.2) Conversely,let $\mathcal{F}$ and $\mathcal{G}$ be semistable vector bundles of rank two on $C$ with the property that $\left(c_{1}(\mathcal{F}), c_{1}(\mathcal{G})\right)=(0,2)$ or $(1,1)$. We put $\mathcal{E}=\rho^{*} \mathcal{G} \otimes H(\mathcal{F})$, where $\rho: \mathbb{P}_{C}(\mathcal{F}) \rightarrow C$ is the bundle map. Then $\mathcal{E}$ is an ample vector bundle on $S:=\mathbb{P}_{C}(\mathcal{F})$ and a polarized surface $(S, \operatorname{det} E)$ satisfies the condition of $(2.3 ; \mathrm{V}-1)$.

To see this,the ampleness of $\mathcal{E}$ is the only non-trivial part. Let $F_{1}$ be any fiber of $\rho: \mathbb{P}_{C}(\mathcal{F}) \rightarrow C$ and $F_{2}$ any fiber of $\mathbb{P}_{C}(\mathcal{G}) \rightarrow C$. By the semistability criterion in $[\mathrm{Mi} ;(3.1)], 2 H(\mathcal{F})-e F_{1}$ and $2 H(\mathcal{G})-(2-e) F_{2}$ are nef, where $e=c_{1}(\mathcal{F})$. Since $M:=\mathbb{P}_{S}(\mathcal{E}) \simeq S \times_{C} \mathbb{P}(\mathcal{G})$ and $H(\mathcal{E})=[H(\mathcal{F})]_{M}+[H(\mathcal{G})]_{M}$, for the fiber $F:=\left[F_{1}\right]_{M}=\left[F_{2}\right]_{M}$ of the morphism $M \rightarrow C, 2 H(\mathcal{E})-2 F=\left[2 H(\mathcal{F})-e F_{1}\right]_{M}+$ $\left[2 H(\mathcal{G})-(2-e) F_{2}\right]_{M}$ is nef on $M$. Hence $H(\mathcal{E})$ is ample and then $\mathcal{E}$ is ample.

(2.8) Suppose that $(S, A)$ is of the type $(2.3 ; \mathrm{V}-2)$. Our argument is similar to (2.7). For any fiber $F$ of the bundle map $\rho: \mathbb{P}_{C}(\mathcal{F}) \rightarrow C$, we have $F \simeq \mathbb{P}^{1}$ and $A F=3$, hence there are only two possibilities:

a) rank $\mathcal{E}=3$ and $\mathcal{E}_{F}=\mathcal{O}(1)^{\oplus 3}$; b) rank $\mathcal{E}=2$ and $\mathcal{E}_{F}=\mathcal{O}(1) \oplus \mathcal{O}(2)$.

(2.8.1) In the case $(2.8 ; a), \mathcal{G}:=\rho_{*}(\mathcal{E} \otimes[-H(\mathcal{F})])$ is a locally free sheaf of rank three on $C$. Moreover we have

$$
\mathcal{E} \simeq \rho^{*} \mathcal{G} \otimes H(\mathcal{F}), M=\mathbb{P}_{S}(\mathcal{E}) \simeq S \times_{C} \mathbb{P}(\mathcal{F}), c_{1}(\mathcal{G})=1, c_{2}(\mathcal{E})=2, L^{4}=4,
$$

$\mathcal{F}$ is semistable and $\mathcal{G}$ is stable.

Conversely,let $\mathcal{F}$ and $\mathcal{G}$ be semistable vector bundles on $C$ with the property that $\operatorname{rank} \mathcal{F}=2, c_{1}(\mathcal{F})=0$, rank $\mathcal{G}=3, c_{1}(\mathcal{G})=1$. We put $S=\mathbb{P}_{C}(\mathcal{F})$ and $\mathcal{E}=$ 
(2.8.2) In the case $(2.8 ; \mathrm{b}), \mathcal{G}:=\rho_{*}(\mathcal{E} \otimes[-2 H(\mathcal{F})])$ is an invertible sheaf on $C$. Using a natural map $\rho^{*} \mathcal{G} \rightarrow \mathcal{E} \otimes[-2 H(\mathcal{F})]$, we obtain an exact sequence $0 \rightarrow$ $\rho^{*} \mathcal{G} \otimes[2 H(\mathcal{F})] \rightarrow \mathcal{E} \rightarrow Q \rightarrow 0$ for some line bundle $Q$ on $S$. Since $\operatorname{det} \mathcal{E}=$ $2 H(\mathcal{F})+\rho^{*} \mathcal{G}+Q$, we have $Q=H(\mathcal{F})+\rho^{*} T$ for some line bundle $T$ on $C$ with $\operatorname{deg} \mathcal{G}+\operatorname{deg} T=1$. $Q$ is ample since it is a quotient bundle of $\mathcal{E}$, hence we have $\operatorname{deg} T>0$. On the other hand $c_{2}(\mathcal{E})=c_{1}\left(\rho^{*} \mathcal{G}+2 H(\mathcal{F}) c_{1}\left(H(\mathcal{F})+\rho^{*} T\right)=1+\operatorname{deg} T\right.$, thus we have $\operatorname{deg} T<5$ from (2.4). Hence there are only the following possibilities:
a) $\operatorname{deg} T=1, \operatorname{deg} \mathcal{G}=0, \quad c_{2}(\mathcal{E})=2$, and $L^{3}=4$;
b) $\operatorname{deg} T=2, \operatorname{deg} \mathcal{G}=-1, c_{2}(\mathcal{E})=3$, and $L^{3}=3$;
c) $\operatorname{deg} T=3, \operatorname{deg} \mathcal{G}=-2, c_{2}(\mathcal{E})=4$, and $L^{3}=2$;
d) $\operatorname{deg} T=4, \operatorname{deg} \mathcal{G}=-3, c_{2}(\mathcal{E})=5$, and $L^{3}=1$.

(2.9) Suppose that $(S, A)$ is of the type $(2.3 ; \mathrm{VI})$. Our results are similar to [BiLL;(1.4.2)]. Since $A l=4$ for any line $l$ on $\mathbb{P}^{2}$,we have rank $\mathcal{E} \leq 4$.

(2.9.1) When rank $\mathcal{E}=4$, we can prove that $\mathcal{E} \simeq \mathcal{O}(1)^{\oplus 4}$ by similar argument as in [V]; see [OSS;Chapter I,(3.2.1)] for a proof.

(2.9.2) When rank $\mathcal{E}=3, \mathcal{E}$ is a uniform vector bundle on $\mathbb{P}^{2}$, thus the result [E] applies. In particular,we have $\mathcal{E} \simeq \mathcal{O}(1)^{\oplus 2} \oplus \mathcal{O}(2)$ or $T_{\mathbb{P}^{2}} \oplus \mathcal{O}(1)$, where $T_{\mathbb{P}^{2}}$ is the tangent bundle of $\mathbb{P}^{2}$.

(2.9.3) When $\operatorname{rank} \mathcal{E}=2$ and $\mathcal{E}$ is a Fano bundle (i.e. the anti-canonical bundle $-K^{\mathbb{P}(\mathcal{E})}$ of $\mathbb{P}(\mathcal{E})$ is ample), the theorem in $[\mathrm{SW}]$ applies and $\mathcal{E}$ is one of the following types.

a) $\mathcal{E} \simeq \mathcal{O}(1) \oplus \mathcal{O}(3)$ or $\mathcal{O}(2) \oplus \mathcal{O}(2)$.

b) There is an exact sequence $0 \rightarrow \mathcal{O}(2) \rightarrow \mathcal{E} \rightarrow \mathcal{I}_{x}(2) \rightarrow 0$, where $\mathcal{I}_{x}$ is the ideal sheaf of one point $x \in \mathbb{P}^{2}$.

c) $\mathcal{E}$ is stable with $c_{2}(\mathcal{E})=6, \mathcal{E}(-1)$ is spanned, and $0 \rightarrow \mathcal{O}(-1)^{\oplus 2} \rightarrow \mathcal{O}^{\oplus 4} \rightarrow$ $\mathcal{E}(-1) \rightarrow 0$ is exact.

d) $\mathcal{E}$ is stable with $c_{2}(\mathcal{E})=7, \mathcal{E}(-1)$ is spanned,and $0 \rightarrow \mathcal{O}(-2) \rightarrow \mathcal{O}^{\oplus 3} \rightarrow$ $\mathcal{E}(-1) \rightarrow 0$ is exact.

Even in the case that $\mathcal{E}$ is not a Fano bundle, we can apply the argument in [SW]. As a result, $\mathcal{E}$ is of the type b) above if $\mathcal{E}$ is not stable; $7 \leq c_{2}(\mathcal{E}) \leq 15$ if $\mathcal{E}$ is stable.

(2.10) Suppose that $(S, A)$ is of the type (2.3;VII-1). Since $A F=2$ for every fiber $F$ of $\rho: \Sigma_{e} \rightarrow \mathbb{P}^{1}$, we have rank $\mathcal{E}=2$ and $\mathcal{E}_{F}=\mathcal{O}(1) \oplus \mathcal{O}(1)$. Then $\mathcal{G}:=\rho_{*}(\mathcal{E} \otimes[-H])$ is a locally free sheaf of rank two on $\mathbb{P}^{1}$ and $\rho^{*} \mathcal{G} \simeq \mathcal{E} \otimes[-H]$. Let $Z$ be the section of $\rho$ corresponding to a natural surjection $\mathcal{O}_{\mathbb{P}^{1}} \oplus \mathcal{O}_{\mathbb{P}^{1}}(e) \rightarrow \mathcal{O}_{\mathbb{P}^{1}}$. Then $H_{Z}=\mathcal{O}_{Z}$ and $\left[\rho^{*} \mathcal{G}\right]_{Z} \simeq \mathcal{E}_{Z}$. Hence $\mathcal{G}$ is ample and we obtain

a) $\mathcal{E} \simeq\left[H+H_{\xi}\right] \oplus\left[H+3 H_{\xi}\right]$ or $\left[H+2 H_{\xi}\right]^{\oplus 2}$ when $e=0$;

b) $\mathcal{E} \simeq\left[H+H_{\xi}\right] \oplus\left[H+2 H_{\xi}\right]$ when $e=1$;

c) $\mathcal{E} \simeq\left[H+H_{\xi}\right]^{\oplus 2}$ when $e=2$.

In these cases, $c_{2}(\mathcal{E})=4$ and $L^{3}=12$.

\section{$\S 3$ The case of a hyperquadric fibration over a curve.}

In this section we study the case (3) of the theorem (1.5), following the idea in [F7; 33$]$.

(3.1) Since $h^{0}\left(F, L_{F}\right)=n+1$ for every fiber $F$ of $\Phi, \mathcal{E}:=\Phi_{*} \mathcal{O}_{M}[L]$ is a locally 
a $C$-morphism $\rho: M \rightarrow \mathbb{P}_{C}(\mathcal{E})$ and for every point $x$ on $C$ the restriction of $\rho$ to $F_{x}:=\Phi^{-1}(x)$ is an embedding of $F_{x}$ into $\mathbb{P}^{n}$. Hence $\rho$ itself is an embedding and $M$ is a member of $\left|2 H(\mathcal{E})+B_{\mathbb{P}(\mathcal{E})}\right|$ for some line bundle $B$ on $C$. We put $d=L^{n}, e=c_{1}(\mathcal{E}), b=\operatorname{deg} B$ and denote by $g(C)$ the genus of $C$. After simple computation, we get $d=2 e+b, 2 g(C)+e+b=4$, and $s:=2 e+(n+1) b \geq 0$. Furthermore in the last inequality,equality holds if and only if every fiber of $\rho$ is smooth. From these results, we have $(n+1) d+s+4 n g(C)=8 n$, hence $g(C)=0$ or 1 .

(3.2) We first study the case $g(C)=1$. In this case, $C$ is an elliptic curve and we have $e=d-2$ and $b=4-d$ from the equality above. Hence we obtain $d \leq 6$ since $s \geq 0$ and $n \geq 3$.

(3.3) We study the ampleness of $\mathcal{E}$. If $\mathcal{E}$ is ample,then $\operatorname{det} \mathcal{E}$ is ample and $e=$ $c_{1}(\mathcal{E})>0$. It follows that $d>2$, hence $\mathcal{E}$ is not ample when $d \leq 2$. On the other hand, $\mathcal{E}$ is ample when $d \geq 5$ by the argument in [F7;(3.13)]. In general,for any indecomposable vector bundle $\mathcal{F}$ on an elliptic curve, $\mathcal{F}$ is ample if and only if $c_{1}(\mathcal{F})>0$ (for a proof,see e.g. [H1]). Thus when $d=3$ or $4, \mathcal{E}$ is ample if it is indecomposable.

(3.4) When $d=3$ or 4 , we can find an example of $(M, L)$ by the argument in [F7;(3.12)]. We can also find an example of $(M, L)$ with $d=6$ as follows. Let $C$ be a smooth elliptic curve and take a line bundle $\mathcal{L}$ on $C$ with $\operatorname{deg} \mathcal{L}=1$. We put $\mathcal{E}=\mathcal{L}^{\oplus 4}$,then $\mathcal{E}$ is ample, $c_{1}(\mathcal{E})=4, \mathbb{P}_{C}(\mathcal{E}) \simeq C \times \mathbb{P}_{\sigma}^{3}$, and $H(\mathcal{E})=H_{\sigma}+\mathcal{L}_{\mathbb{P}(\mathcal{E})}$, where $H_{\sigma}$ is the pullback of $\mathcal{O}(1)$ on $\mathbb{P}_{\sigma}^{3}$. Putting $B=-2 \mathcal{L}$, we have $\operatorname{deg} B=-2$ and $2 H(\mathcal{E})+B_{\mathbb{P}(\mathcal{E})}=2 H_{\sigma}$. Then a general member $M$ of $\left|2 H(\mathcal{E})+B_{\mathbb{P}(\mathcal{E})}\right|$ is smooth and,putting $L=[H(\mathcal{E})]_{M},(M, L)$ becomes an expected example with $d=6$.

(3.5) From now on,we study the case $g(C)=0$. In this case, $C \simeq \mathbb{P}_{\xi}^{1}$ and we have $e=d-4$ and $b=8-d$ from the equality in (3.1). Hence we obtain $d \leq 12$ since $s \geq 0$ and $n \geq 3$. Furthermore when $d=11$ or 12 ,we have $n=3$ and when $d=12$, we have $s=0$ and $\Phi$ is a $\mathbb{P}^{1} \times \mathbb{P}^{1}$-bundle over $\mathbb{P}_{\xi}^{1}$ by $[\mathrm{F} 7 ;(3.3)]$.

(3.6) We put $P=\mathbb{P}_{C}(\mathcal{E}), H=H(\mathcal{E})$ and $H_{\xi}=\pi^{*} \mathcal{O}_{C}(1)$, where $\pi$ is the bundle $\operatorname{map} \mathbb{P}_{C}(\mathcal{E}) \rightarrow C$. Since $\mathcal{E}$ is decomposable, we can describe $\mathcal{E} \simeq \mathcal{O}\left(e_{0}\right) \oplus \cdots \oplus \mathcal{O}\left(e_{n}\right)$, where $e_{0}, \ldots, e_{n} \in \mathbb{Z}, e_{0} \leq \cdots \leq e_{n}$, and $\sum_{i=0}^{n} e_{i}=e . \mathcal{O}\left(e_{0}\right) \oplus \cdots \oplus \mathcal{O}\left(e_{n}\right)$ is denoted by $\mathcal{O}\left(e_{0}, \ldots, e_{n}\right)$ for simplicity. We shall classify $\mathcal{E} \simeq \mathcal{O}\left(e_{0}, \ldots, e_{n}\right)$ for each case $d=1,2, \ldots, 12$.

(3.7)Lemma. $2\left(e_{n-1}+e_{n}\right)<d$ when $e_{0} \leq 0$.

Proof. (cf. $[\mathrm{F} 7 ;(3.24)])$. A natural surjection $\mathcal{E} \rightarrow \mathcal{O}\left(e_{0}, \ldots, e_{n-1}\right)$ gives a prime divisor $D_{1}:=\mathbb{P}\left(\mathcal{O}\left(e_{0}, \ldots, e_{n-1}\right)\right)$ on $P$. Similarly $\mathcal{E} \rightarrow \mathcal{O}\left(e_{0}, \ldots, e_{n-2}, e_{n}\right)$ gives a prime divisor $D_{2}:=\mathbb{P}\left(\mathcal{O}\left(e_{0}, \ldots, e_{n-2}, e_{n}\right)\right)$ on $P$ and $\mathcal{E} \rightarrow \mathcal{O}\left(e_{0}, \ldots, e_{n-2}\right)$ gives a subvariety $W:=\mathbb{P}\left(\mathcal{O}\left(e_{0}, \ldots, e_{n-2}\right)\right)$ on $P$. We have $D_{1} \in\left|H-e_{n} H_{\xi}\right|, D_{2} \in$ $\left|H-e_{n-1} H_{\xi}\right|$, and $W=D_{1} \cap D_{2}$ as schemes. When $e_{0} \leq 0$, we have $W \not \subset M$ since $H_{W}$ is not ample. Hence $\operatorname{dim}(M \cap W)=n-2$ and $0<L^{n-2}\{M \cap W\}=$ $H^{n-2}\left(2 H+b H_{\xi}\right)\left(H-e_{n} H_{\xi}\right)\left(H-e_{n-1} H_{\xi}\right)=d-2\left(e_{n-1}+e_{n}\right)$.

(3.8) Suppose that $d=1$. We have $e=-3, b=7$, and $M \in\left|2 H+7 H_{\xi}\right|$. By $(3.7), \mathcal{E} \simeq \mathcal{O}(-3,0, \ldots, 0), \mathcal{O}(-2,-1,0, \ldots, 0)$, or $\mathcal{O}(-1,-1,-1,0, \ldots, 0)$.

(3.8.1) When $\mathcal{E} \simeq \mathcal{O}(-1,-1,-1,0, \ldots, 0)$, we have $n \leq 4$ by the argument in $[\mathrm{F} 7 ;(3.21)]$. Indeed,we have

$$
P \simeq\left\{\left(\xi_{0}: \xi_{1}\right) \times\left(\sigma_{0}: \sigma_{1}: \sigma_{2}: \sigma_{30}: \sigma_{31}: \cdots: \sigma_{n 0}: \sigma_{n 1}\right) \in \mathbb{P}_{\xi}^{1} \times \mathbb{P}_{\sigma}^{2 n-2}\right\}
$$


,H $H=H_{\sigma}-H_{\xi}$ and $M \in\left|2 H_{\sigma}+5 H_{\xi}\right|$. Thus we can describe

$$
M=\left\{q_{0}(\sigma) \xi_{0}^{5}+q_{1}(\sigma) \xi_{0}^{4} \xi_{1}+\cdots+q_{5}(\sigma) \xi_{1}^{5}=0 \text { in } P\right\}
$$

,where $q_{0}, \ldots, q_{5}$ are homogeneous polynomials of degree two in $\sigma_{0}, \sigma_{1}, \ldots, \sigma_{n 1}$. In this defining equation of $M$, we put

$$
\begin{aligned}
& \sigma_{0}=a_{00} \xi_{0}+a_{01} \xi_{1}, \sigma_{1}=a_{10} \xi_{0}+a_{11} \xi_{1}, \sigma_{2}=a_{20} \xi_{0}+a_{21} \xi_{1} \\
& \sigma_{30}=a_{3} \xi_{0}, \sigma_{31}=a_{3} \xi_{1}, \ldots, \sigma_{n 0}=a_{n} \xi_{0}, \sigma_{n 1}=a_{n} \xi_{1}
\end{aligned}
$$

,where $a_{00}, a_{01}, \ldots, a_{n}$ are constants. Then we obtain an equation

$$
Q_{0}(a) \xi_{0}^{7}+Q_{1}(a) \xi_{0}^{6} \xi_{1}+\cdots+Q_{7}(a) \xi_{1}^{7}=0
$$

,where $Q_{0}, \ldots, Q_{7}$ are homogeneous polynomials of degree two in $(a)=\left(a_{00}, a_{01}, \ldots, a_{n}\right)$. If $n \geq 5$, then $Q_{0}(a)=\cdots=Q_{7}(a)=0$ has a non-trivial solution. We fix such a solution $(a)$ and define a rational map $\alpha: \mathbb{P}_{\xi}^{1} \rightarrow \mathbb{P}_{\sigma}^{2 n-2}$ by

$$
\begin{gathered}
\alpha\left(\xi_{0}: \xi_{1}\right):=\left(a_{00} \xi_{0}+a_{01} \xi_{1}: a_{10} \xi_{0}+a_{11} \xi_{1}: a_{20} \xi_{0}+a_{21} \xi_{1}:\right. \\
\left.: a_{3} \xi_{0}: a_{3} \xi_{1}: \cdots: a_{n} \xi_{0}: a_{n} \xi_{1}\right)
\end{gathered}
$$

If $\alpha$ is not a morphism, then $a_{00}: a_{10}: a_{20}=a_{01}: a_{11}: a_{21}$ and $a_{3}=\cdots=a_{n}=0$. Since $(a)$ is non-trivial,the equations

$$
\sigma_{0}: \sigma_{1}: \sigma_{2}=a_{00}: a_{10}: a_{20}=a_{01}: a_{11}: a_{21}, \sigma_{30}=\sigma_{31}=\cdots=\sigma_{n 0}=\sigma_{n 1}=0
$$

determine a point $z$ on $\mathbb{P}^{2 n-2}$. Let $Z$ be the fiber of a projection $\mathbb{P}_{\xi}^{1} \times \mathbb{P}_{\sigma}^{2 n-2} \rightarrow \mathbb{P}_{\sigma}^{2 n-2}$ over $z$. Then we have $Z \subset M$ by the definition of $Z$, hence $0<L Z=H Z=$ $\left(H_{\sigma}-H_{\xi}\right) Z=-1$. This is a contradiction, thus $\alpha$ is a morphism. Let $\Gamma$ be the graph of $\alpha$. Then $\Gamma \subset M$ by the definition of $\alpha$, hence $0<L \Gamma=H \Gamma=$ $\left(H_{\sigma}-H_{\xi}\right) \Gamma$. However,since $H_{\sigma} \Gamma=H_{\xi} \Gamma=1$, this is also a contradiction. Hence we have proved that $n \leq 4$, thus $\mathcal{E} \simeq \mathcal{O}(-1,-1,-1,0)$ or $\mathcal{O}(-1,-1,-1,0,0)$. If $\mathcal{E} \simeq \mathcal{O}(-1,-1,-1,0)$, then $P \simeq\left\{\left(\xi_{0}: \xi_{1}\right) \times\left(\sigma_{0}: \sigma_{1}: \sigma_{2}: \sigma_{30}: \sigma_{31}\right) \in \mathbb{P}_{\xi}^{1} \times \mathbb{P}_{\sigma}^{4} \mid \xi_{0}:\right.$ $\left.\xi_{1}=\sigma_{30}: \sigma_{31}\right\}$. Thus the projection $\mu: P \rightarrow \mathbb{P}_{\sigma}^{4}$ is the blowing-up of $\mathbb{P}_{\sigma}^{4}$ with center $W:=\left\{\sigma_{30}=\sigma_{31}=0\right.$ in $\left.\mathbb{P}_{\sigma}^{4}\right\} \simeq \mathbb{P}^{2}$. Since the exceptional divisor $E$ of $\mu$ is a member of $\left|H_{\sigma}-H_{\xi}\right|$, we have $M \in\left|7 H_{\sigma}-5 E\right|$. Hence $M$ is the strict transform of a hypersurface of degree seven in $\mathbb{P}_{\sigma}^{4}$, which has singularities with multiplicity five along $W$.

(3.8.2) When $\mathcal{E} \simeq \mathcal{O}(-2,-1,0, \ldots, 0)$, we claim that $n \leq 4$. The following argument is similar to $(3.8 .1)$. We have

$P \simeq\left\{\begin{array}{c}\left(\xi_{0}: \xi_{1}\right) \times\left(\sigma_{0}: \sigma_{10}: \sigma_{11}: \sigma_{20}: \sigma_{21}: \sigma_{22}: \cdots: \sigma_{n 0}: \sigma_{n 1}: \sigma_{n 2}\right) \in \mathbb{P}_{\xi}^{1} \times \mathbb{P}_{\sigma}^{3 n-1} \\ \mid \xi_{0}: \xi_{1}=\sigma_{10}: \sigma_{11}=\sigma_{20}: \sigma_{21}=\sigma_{21}: \sigma_{22}=\cdots=\sigma_{n 0}: \sigma_{n 1}=\sigma_{n 1}: \sigma_{n 2}\end{array}\right\}$

,H $H=H_{\sigma}-2 H_{\xi}$ and $M \in\left|2 H_{\sigma}+3 H_{\xi}\right|$. Thus $M=\left\{q_{0}(\sigma) \xi_{0}^{3}+q_{1}(\sigma) \xi_{0}^{2} \xi_{1}+q_{2}(\sigma) \xi_{0} \xi_{1}^{2}+\right.$ $q_{3}(\sigma) \xi_{1}^{3}=0$ in $\left.P\right\}$, where $q_{0}, \ldots, q_{3}$ are quadric polynomials in $(\sigma)$. We put

$$
\sigma_{0}=a_{00} \xi_{0}^{2}+a_{01} \xi_{0} \xi_{1}+a_{02} \xi_{1}^{2}, \sigma_{10}=\xi_{0}\left(a_{10} \xi_{0}+a_{11} \xi_{1}\right), \sigma_{11}=\xi_{1}\left(a_{10} \xi_{0}+a_{11} \xi_{1}\right)
$$


Then from the defining equation of $M$ above,we obtain an equation

$$
Q_{0}(a) \xi_{0}^{7}+Q_{1}(a) \xi_{0}^{6} \xi_{1}+\cdots+Q_{7}(a) \xi_{1}^{7}=0
$$

, where $Q_{0}, \ldots, Q_{7}$ are quadric polynomials in $(a)=\left(a_{00}, a_{01}, \ldots, a_{n}\right)$. If $n \geq 5$, then $Q_{0}(a)=\cdots=Q_{7}(a)=0$ has a non-trivial solution $(a)$. We fix it and define a rational map $\alpha: \mathbb{P}_{\xi}^{1} \rightarrow \mathbb{P}_{\sigma}^{3 n-1}$ by

$$
\begin{gathered}
\alpha\left(\xi_{0}: \xi_{1}\right):=\left(a_{00} \xi_{0}^{2}+a_{01} \xi_{0} \xi_{1}+a_{02} \xi_{1}^{2}: \xi_{0}\left(a_{10} \xi_{0}+a_{11} \xi_{1}\right): \xi_{1}\left(a_{10} \xi_{0}+a_{11} \xi_{1}\right): a_{2} \xi_{0}^{2}\right. \\
\left.: a_{2} \xi_{0} \xi_{1}: a_{2} \xi_{1}^{2}: \cdots: a_{n} \xi_{0}^{2}: a_{n} \xi_{0} \xi_{1}: a_{n} \xi_{1}^{2}\right)
\end{gathered}
$$

If $\alpha$ is not a morphism, then $a_{2}=\cdots=a_{n}=0$ and for some $\left(c_{0}: c_{1}\right) \in \mathbb{P}_{\xi}^{1}$, we have $a_{10} c_{0}+a_{11} c_{1}=0$ and $a_{00} c_{0}^{2}+a_{01} c_{0} c_{1}+a_{02} c_{1}^{2}=0$. In the case $a_{10}=a_{11}=0$,let $Z$ be the fiber of $\mathbb{P}_{\xi}^{1} \times \mathbb{P}_{\sigma}^{3 n-1} \rightarrow \mathbb{P}_{\sigma}^{3 n-1}$ over $z:=(1: 0: \cdots: 0)$. Then we have $Z \subset M$, hence $0<L Z=H Z=\left(H_{\sigma}-2 H_{\xi}\right) Z=-2$. This is a contradiction,thus $a_{10} \neq 0$ or $a_{11} \neq 0$.

In this case, $a_{00} \xi_{0}^{2}+a_{01} \xi_{0} \xi_{1}+a_{02} \xi_{1}^{2}$ is devided by $a_{10} \xi_{0}+a_{11} \xi_{1}$ in $\mathbb{C}\left[\xi_{0}, \xi_{1}\right]$; we denote by $b_{0} \xi_{0}+b_{1} \xi_{1}$ its quotient. We put

$$
Z=\left\{\sigma_{0}=b_{0} \sigma_{10}+b_{1} \sigma_{11}, \sigma_{20}=\cdots=\sigma_{n 2}=0 \text { in } P\right\} .
$$

Then $\operatorname{dim} Z=1$ and $Z \subset M$ by the definition of $Z$, hence $0<L Z=H Z=$ $\left(H_{\sigma}-2 H_{\xi}\right) Z$. However, since $H_{\sigma} Z=1$ and $H_{\xi} Z=1$, this is a contradiction too. Thus $\alpha$ is a morphism.

Let $\Gamma$ be the graph of $\alpha$. We have $\Gamma \subset M$ and then $0<L \Gamma=H \Gamma=\left(H_{\sigma}-\right.$ $\left.2 H_{\xi}\right) \Gamma$. However,since $H_{\sigma} \Gamma=2$ and $H_{\xi} \Gamma=1$, this is also a contradiction. Hence we have proved that $n \leq 4$, thus $\mathcal{E} \simeq \mathcal{O}(-2,-1,0,0)$ or $\mathcal{O}(-2,-1,0,0,0)$.

(3.8.3) When $\mathcal{E} \simeq \mathcal{O}(-3,0, \ldots, 0)$, we claim that $n \leq 4$ as before. $P$ is isomorphic to

$\left\{\begin{array}{c}\left(\xi_{0}: \xi_{1}\right) \times\left(\sigma_{0}: \sigma_{10}: \sigma_{11}: \sigma_{12}: \sigma_{13}: \cdots: \sigma_{n 0}: \sigma_{n 1}: \sigma_{n 2}: \sigma_{n 3}\right) \in \mathbb{P}_{\xi}^{1} \times \mathbb{P}_{\sigma}^{4 n} \\ \mid \xi_{0}: \xi_{1}=\sigma_{10}: \sigma_{11}=\sigma_{11}: \sigma_{12}=\sigma_{12}: \sigma_{13}=\cdots=\sigma_{n 0}: \sigma_{n 1}=\sigma_{n 1}: \sigma_{n 2}=\sigma_{n 2}: \sigma_{n 3}\end{array}\right\}$

,H $H=H_{\sigma}-3 H_{\xi}$ and $M \in\left|2 H_{\sigma}+H_{\xi}\right|$. Thus $M=\left\{q_{0}(\sigma) \xi_{0}+q_{1}(\sigma) \xi_{1}=0\right.$ in $\left.P\right\}$, where $q_{0}$ and $q_{1}$ are quadric polynomials in $(\sigma)$. We put

$$
\begin{aligned}
& \sigma_{0}=a_{00} \xi_{0}^{3}+a_{01} \xi_{0}^{2} \xi_{1}+a_{02} \xi_{0} \xi_{1}^{2}+a_{03} \xi_{1}^{3}, \\
& \sigma_{10}=a_{1} \xi_{0}^{3}, \sigma_{11}=a_{1} \xi_{0}^{2} \xi_{1}, \sigma_{12}=a_{1} \xi_{0} \xi_{1}^{2}, \sigma_{13}=a_{1} \xi_{1}^{3}, \ldots \\
& \sigma_{n 0}=a_{n} \xi_{0}^{3}, \sigma_{n 1}=a_{n} \xi_{0}^{2} \xi_{1}, \sigma_{n 2}=a_{n} \xi_{0} \xi_{1}^{2}, \sigma_{n 3}=a_{n} \xi_{1}^{3} .
\end{aligned}
$$

Then from the defining equation of $M$ above,we obtain an equation

$$
Q_{0}(a) \xi_{0}^{7}+Q_{1}(a) \xi_{0}^{6} \xi_{1}+\cdots+Q_{7}(a) \xi_{1}^{7}=0
$$

, where $Q_{0}, \ldots, Q_{7}$ are quadric polynomials in $(a)=\left(a_{00}, a_{01}, \ldots, a_{n}\right)$. If $n \geq 5$, then $Q_{0}(a)=\cdots=Q_{7}(a)=0$ has a non-trivial solution $(a)$. We fix it and define a rational map $\alpha: \mathbb{P}_{\xi}^{1} \rightarrow \mathbb{P}_{\sigma}^{4 n}$ by

$$
\alpha\left(\xi_{0}: \xi_{1}\right):=\left(a_{00} \xi_{0}^{3}+a_{01} \xi_{0}^{2} \xi_{1}+a_{02} \xi_{0} \xi_{1}^{2}+a_{03} \xi_{1}^{3}: a_{1} \xi_{0}^{3}: a_{1} \xi_{0}^{2} \xi_{1}: a_{1} \xi_{0} \xi_{1}^{2}: a_{1} \xi_{1}^{3}\right.
$$


If $\alpha$ is not a morphism, then $a_{1}=\cdots=a_{n}=0$. Let $Z$ be the fiber of $\mathbb{P}_{\xi}^{1} \times \mathbb{P}_{\sigma}^{4 n} \rightarrow \mathbb{P}_{\sigma}^{4 n}$ over $z:=(1: 0: \cdots: 0)$. We have $Z \subset M$ and then $0<L Z=H Z=\left(H_{\sigma}-\right.$ $\left.3 H_{\xi}\right) Z=-3$. This is a contradiction,hence $\alpha$ is a morphism. Let $\Gamma$ be the graph of $\alpha$. We have $\Gamma \subset M$ and then $0<L \Gamma=H \Gamma=\left(H_{\sigma}-3 H_{\xi}\right) \Gamma$. However,since $H_{\sigma} \Gamma=3$ and $H_{\xi} \Gamma=1$, this is also a contradiction. Hence we have proved that $n \leq 4$, thus $\mathcal{E} \simeq \mathcal{O}(-3,0,0,0)$ or $\mathcal{O}(-3,0,0,0,0)$.

(3.9) Now we study the case $d=2$. We have $e=-2, b=6$, and $M \in\left|2 H+6 H_{\xi}\right|$. By $(3.7), \mathcal{E} \simeq \mathcal{O}(-2,0, \ldots, 0)$ or $\mathcal{O}(-1,-1,0, \ldots, 0)$.

(3.9.1) When $\mathcal{E} \simeq \mathcal{O}(-1,-1,0, \ldots, 0)$, we have $n \leq 4$ as in (3.8.1). Hence $\mathcal{E} \simeq \mathcal{O}(-1,-1,0,0)$ or $\mathcal{O}(-1,-1,0,0,0)$

(3.9.2) When $\mathcal{E} \simeq \mathcal{O}(-2,0, \ldots, 0)$,we have $n \leq 4$ as in (3.8.2). Hence $\mathcal{E} \simeq$ $\mathcal{O}(-2,0,0,0)$ or $\mathcal{O}(-2,0,0,0,0)$.

(3.10) Suppose that $d=3$. Then $e=-1, b=5$, and $M \in\left|2 H+5 H_{\xi}\right|$. From $(3.7)$, we have $\mathcal{E} \simeq \mathcal{O}(-2,0, \ldots, 0,1), \mathcal{E} \simeq \mathcal{O}(-1,-1,0, \ldots, 0,1)$, or $\mathcal{E} \simeq$ $\mathcal{O}(-1,0, \ldots, 0)$.

(3.10.1) When $\mathcal{E} \simeq \mathcal{O}(-1,0, \ldots, 0)$, we have $n \leq 4$ as in (3.8.1). Hence $\mathcal{E} \simeq$ $\mathcal{O}(-1,0,0,0)$ or $\mathcal{O}(-1,0,0,0,0)$.

(3.10.2) When $\mathcal{E} \simeq \mathcal{O}(-1,-1,0, \ldots, 0,1)$, we have $n \leq 4$ by the argument in $[\mathrm{F} 7 ;(3.23 .2)]$ which is similar to (3.8.1). Hence $\mathcal{E} \simeq \mathcal{O}(-1,-1,0,1)$ or $\mathcal{O}(-1,-1,0,0,1)$.

(3.10.3) When $\mathcal{E} \simeq \mathcal{O}(-2,0, \ldots, 0,1)$, we have $n \leq 4$ as in (3.8.2) and (3.10.2). Hence $\mathcal{E} \simeq \mathcal{O}(-2,0,0,1)$ or $\mathcal{O}(-2,0,0,0,1)$.

The next lemma is useful for $d \geq 4$.

(3.11)Lemma. When $d \geq 4,-1$ does not appear twice in $\left\{e_{0}, \ldots, e_{n}\right\}$.

We can prove this lemma by the argument in $[\mathrm{F} 7 ;(3.18)]$.

(3.12) Now we study the case $d=4$. We have $e=0, b=4$, and $M \in\left|2 H+4 H_{\xi}\right|$. By $(3.7)$ and $(3.11), \mathcal{E} \simeq \mathcal{O}(-1,0, \ldots, 0,1)$ or $\mathcal{O}(0, \ldots, 0)$.

(3.12.1) When $\mathcal{E} \simeq \mathcal{O}(-1,0, \ldots, 0,1)$, we have $n \leq 4$ as in (3.10.2). Hence $\mathcal{E} \simeq \mathcal{O}(-1,0,0,1)$ or $\mathcal{O}(-1,0,0,0,1)$.

(3.12.2) When $\mathcal{E} \simeq \mathcal{O}(0, \ldots, 0)$, by the argument in $[\mathrm{F} 7 ;(3.23 .1)]$, we have $n \leq$ $4, P \simeq \mathbb{P}_{\xi}^{1} \times \mathbb{P}_{\sigma}^{n}, \mathrm{Bs}|L|=\phi$, and the morphism $\varphi: M \rightarrow \mathbb{P}_{\sigma}^{n}$ defined by $|L|$ is a finite morphism of degree four. Conversely,any general member $M$ of $\left|2 H_{\sigma}+4 H_{\xi}\right|$ on $P$ does not contain any fiber of the projection $P \rightarrow \mathbb{P}_{\sigma}^{n}$, thus $L:=H_{M}$ is ample and $(M, L)$ is a polarized manifold of the above type.

The next lemma is useful for $d \geq 5$.

(3.13)Lemma. $e_{0} \geq-1$ when $d \geq 5$.

We can prove this lemma by the argument in $[\mathrm{F} 7 ;(3.19)]$.

Similarly we obtain the following two lemmas.

(3.14)Lemma. $e_{0} \geq 0$ when $d \geq 7$.

(3.15)Lemma. $e_{0} \geq 1$ when $d \geq 9$.

(3.16) Now we study the case $d=5$. We have $e=1, b=3$, and $M \in\left|2 H+3 H_{\xi}\right|$. By $(3.11)$ and $(3.13), \mathcal{E} \simeq \mathcal{O}(-1,0, \ldots, 0,2), \mathcal{O}(-1,0, \ldots, 0,1,1)$,or $\mathcal{O}(0, \ldots, 0,1)$.

(3.16.1) When $\mathcal{E} \simeq \mathcal{O}(-1,0, \ldots, 0,2)$, we have $n \leq 3$ as in $(3.10 .2)$, hence $\mathcal{E} \simeq$ $\mathcal{O}(-1,0,0,2)$. Furthermore $\mathrm{Bs}|L|$ is one point as in $[\mathrm{F} 7 ;(3.23 .2)]$.

(3.16.2) When $\mathcal{E} \simeq \mathcal{O}(-1,0, \ldots, 0,1,1)$, we have $n \leq 4$ and $\mathrm{Bs}|L|$ is one point as 
(3.16.3) When $\mathcal{E} \simeq \mathcal{O}(0, \ldots, 0,1)$, by the argument in $[\mathrm{F} 7 ;(3.24)]$, we have $n \leq 4$ and $|L|$ makes $M$ the normalization of a hypersurface of degree five in $\mathbb{P}^{n+1}$, which has triple points along a $\mathbb{P}^{2}$ in $\mathbb{P}^{n+1}$.

(3.17) Suppose that $d=6$. We have $e=2, b=2$, and $M \in\left|2 H+2 H_{\xi}\right|$. By $(3.7),(3.11)$, and $(3.13), \mathcal{E} \simeq \mathcal{O}(-1,0, \ldots, 0,1,1,1), \mathcal{O}(0, \ldots, 0,1,1)$,or $\mathcal{O}(0, \ldots, 0,2)$.

(3.17.1) When $\mathcal{E} \simeq \mathcal{O}(-1,0, \ldots, 0,1,1,1)$, we show that $n=3$ similarly as in (3.7). Natural surjections $\mathcal{E} \rightarrow \mathcal{O}\left(e_{0}, \ldots, e_{n-1}\right), \mathcal{E} \rightarrow \mathcal{O}\left(e_{0}, \ldots, e_{n-2}, e_{n}\right)$, and $\mathcal{E} \rightarrow$ $\mathcal{O}\left(e_{0}, \ldots\right.$,

$\left.e_{n-3}, e_{n-1}, e_{n}\right)$ give prime divisors $D_{1}:=\mathbb{P}\left(\mathcal{O}\left(e_{0}, \ldots, e_{n-1}\right)\right), D_{2}:=\mathbb{P}\left(\mathcal{O}\left(e_{0}, \ldots, e_{n-2}, e_{n}\right)\right)$ and $D_{3}:=\mathbb{P}\left(\mathcal{O}\left(e_{0}, \ldots, e_{n-3}, e_{n-1}, e_{n}\right)\right)$ respectively. A natural surjection $\mathcal{E} \rightarrow$ $\mathcal{O}\left(e_{0}, \ldots\right.$,

$\left.e_{n-3}\right)$ gives a subvariety $W:=\left(\mathcal{O}\left(e_{0}, \ldots, e_{n-3}\right)\right)$ of $P=\mathbb{P}(\mathcal{E})$. We have $D_{1} \in$ $\left|H-e_{n} H_{\xi}\right|, D_{2} \in\left|H-e_{n-1} H_{\xi}\right|, D_{3} \in\left|H-e_{n-2} H_{\xi}\right|$, and $W=D_{1} \cap D_{2} \cap D_{3}$ as schemes. Since $H_{W}$ is not ample, we have $W \not \subset M$, hence $\operatorname{dim}(M \cap W)=n-3$ and $0<L^{n-3}\{M \cap W\}=H^{n-3}\left(2 H+2 H_{\xi}\right)\left(H-H_{\xi}\right)^{3}=2 e-4=0$ if $n \leq 4$. This is a contradiction, thus we have $n=3$ and $\mathcal{E} \simeq \mathcal{O}(-1,1,1,1)$. By the argument in $[\mathrm{F} 7 ;(3.26)], M$ is a double covering of $\mathbb{P}_{\xi}^{1} \times \mathbb{P}_{\sigma}^{2}$ and its branch locus is a smooth member of $\left|4 H_{\xi}+2 H_{\sigma}\right|$. We have also $L=\left[H_{\xi}+H_{\sigma}\right]_{M}$.

(3.17.2) When $\mathcal{E} \simeq \mathcal{O}(0, \ldots, 0,1,1)$, we have $n \leq 4$ as in $(3.16 .3)$,hence $\mathcal{E} \simeq$ $\mathcal{O}(0,0,1,1)$ or $\mathcal{O}(0,0,0,1,1)$. We show the existence of $(M, L)$. When $\mathcal{E} \simeq \mathcal{O}(0,0,1,1)$, we have $P \simeq\left\{\left(\xi_{0}: \xi_{1}\right) \times\left(\sigma_{0}: \sigma_{1}: \sigma_{20}: \sigma_{21}: \sigma_{30}: \sigma_{31}\right) \in \mathbb{P}_{\xi}^{1} \times \mathbb{P}_{\sigma}^{5} \mid \xi_{0}: \xi_{1}=\sigma_{20}:\right.$ $\left.\sigma_{21}=\sigma_{30}: \sigma_{31}\right\}$ and $H=H_{\sigma}$. Let $M$ be a general member of $\left|2 H_{\sigma}+2 H_{\xi}\right|$ and put $L=\left[H_{\sigma}\right]_{M}$. Then $\operatorname{Bs}|L|=\phi$ and the restriction of the projection $P \rightarrow \mathbb{P}_{\sigma}^{5}$ to $M$ is the morphism $\varphi$ defined by $|L|$. If $\varphi: M \rightarrow \varphi(M)$ is not finite, $M$ contains a fiber $Z$ of the projection $P \rightarrow \mathbb{P}_{\sigma}^{5}$ over one point $z$ on the line $l:=\left\{\sigma_{20}=\sigma_{21}=\right.$ $\sigma_{30}=\sigma_{31}=0$ in $\left.\mathbb{P}_{\sigma}^{5}\right\}$ Using homogeneous polynomials $q_{0}, q_{1}$, and $q_{2}$ of degree two in $(\sigma)$,we can describe that $M=\left\{q_{0}(\sigma) \xi_{0}^{2}+q_{1}(\sigma) \xi_{0} \xi_{1}+q_{2}(\sigma) \xi_{1}^{2}=0\right.$ in $\left.P\right\}$. Then $Z \subset M$ if and only if $q_{0}(z)=q_{1}(z)=q_{2}(z)=0$. Thus if we choose $q_{0}, q_{1}$, and $q_{2}$ generally to satisfy that $l \cap\left\{q_{0}(\sigma)=q_{1}(\sigma)=q_{2}(\sigma)=0\right.$ in $\left.\mathbb{P}_{\sigma}^{5}\right\}=\phi$, then $\varphi$ becomes finite and $L$ is ample. Similarly we can find examples of $(M, L)$ such that $\mathcal{E} \simeq \mathcal{O}(0,0,0,1,1)$

(3.17.3) When $\mathcal{E} \simeq \mathcal{O}(0, \ldots, 0,2)$, we have $n \leq 3$ as in (3.16.3), hence $\mathcal{E} \simeq$ $\mathcal{O}(0,0,0,2)$. We can show the existence of $(M, L)$ similarly as above.

When $d \geq 7$,the situation is much simpler.

(3.18)Lemma. $B s|L|=\phi$ and $L$ is very ample when $d \geq 7$.

We can prove this lemma similarly as in $[\mathrm{F} 7 ;(3.31)]$. This lemma tells us that our results overlap [I;Theorem 4.3], but our method is different from his.

$(3,19)$ Now we study the case $d=7$. We have $e=3, b=1$, and $M \in\left|2 H+H_{\xi}\right|$. Furthermore $e_{0} \geq 0$ by $(3.14)$ and $e_{2} \geq 1$ by the argument in $[\mathrm{F} 7 ;(3.25)]$. Hence $\mathcal{E} \simeq \mathcal{O}(0,0,1,2), \mathcal{O}(0,1,1,1)$,or $\mathcal{O}(0,0,1,1,1)$. In each case, $(M, L)$ exists similarly as in (3.17.2). By the morphism defined by $|L|, M$ is isomorphic to a manifold of degree seven in $\mathbb{P}^{n+3}$.

(3.20) Suppose that $d=8$. We have $e=4, b=0$, and $M \in|2 H|$. Furthermore $e_{0} \geq 0$ by $(3.14)$ and $e_{1} \geq 1$ by the argument in $[\mathrm{F} 7 ;(3.26)]$. Hence $\mathcal{E} \simeq \mathcal{O}(0,1,1,2), \mathcal{O}(0,1,1,1,1)$,or $\mathcal{O}(1,1,1,1)$

(3.20.1) When $\mathcal{E} \simeq \mathcal{O}(1,1,1,1)$, we have $P \simeq \mathbb{P}_{\xi}^{1} \times \mathbb{P}_{\sigma}^{3}, H=H_{\xi}+H_{\sigma}$, and $M \in$ 
be a general member of $\left|2 H_{\xi}+2 H_{\sigma}\right|$ and put $L=\left[H_{\xi}+H_{\sigma}\right]_{M}$. Since $\mathcal{E}$ is ample, $L$ is ample and $(M, L)$ is a polarized manifold of the above type.

(3.20.2) When $\mathcal{E} \simeq \mathcal{O}(0,1,1,1,1)$, by the argument in $[\mathrm{F} 7 ;(3.26)], M$ is a double covering of $\mathbb{P}_{\xi}^{1} \times \mathbb{P}_{\sigma}^{3}$ and its branch locus is a smooth member of $\left|2 H_{\xi}+2 H_{\sigma}\right|$. We have also $L=\left[H_{\xi}+H_{\sigma}\right]_{M}$.

(3.20.3) Even when $\mathcal{E} \simeq \mathcal{O}(0,1,1,2)$, by the argument in $[\mathrm{F} 7 ;(3.26)]$, we have a morphism $h: M \rightarrow \mathbb{P}_{\xi}^{1} \times \mathbb{P}_{\sigma}^{3}$ and $L=h^{*}\left(H_{\xi}+H_{\sigma}\right)$. Since $L$ is ample, $h: M \rightarrow h(M)$ is finite and $h(M) \in\left|a_{1} H_{\xi}+a_{2} H_{\sigma}\right|$ for some non-negative integers $a_{1}$ and $a_{2}$. Then $8=L^{3}=(\operatorname{deg} h) \cdot\left[H_{\xi}+H_{\sigma}\right]_{h(M)}^{3}=(\operatorname{deg} h)\left(a_{1}+3 a_{2}\right)$. From the construction of $h$, we get $\operatorname{deg} h=2$ and $a_{1}=a_{2}=1$. Hence $h(M) \in\left|H_{\xi}+H_{\sigma}\right|$ and $M \rightarrow h(M)$ is a double covering.

(3.21) Suppose that $d=9$. We have $e=5, b=-1$, and $M \in\left|2 H-H_{\xi}\right|$. Since $e_{0} \geq 1$ by $(3.15), \mathcal{E} \simeq \mathcal{O}(1,1,1,2)$ or $\mathcal{O}(1,1,1,1,1)$.

(3.21.1) When $\mathcal{E} \simeq \mathcal{O}(1,1,1,1,1)$,similarly as in $[\mathrm{F} 7 ;(3.27)]$, the restriction of the projection $P \simeq \mathbb{P}_{\xi}^{1} \times \mathbb{P}_{\sigma}^{4} \rightarrow \mathbb{P}_{\sigma}^{4}$ to $M$ is a blowing-up of $\mathbb{P}_{\sigma}^{4}$ and its center is a complete intersection of two hyperquadrics in $\mathbb{P}_{\sigma}^{4}$.

(3.21.2) When $\mathcal{E} \simeq \mathcal{O}(1,1,1,2)$, we have $P \simeq\left\{\left(\xi_{0}: \xi_{1}\right) \times\left(\sigma_{0}: \sigma_{1}: \sigma_{2}: \sigma_{30}:\right.\right.$ $\left.\left.\sigma_{31}\right) \in \mathbb{P}_{\xi}^{1} \times \mathbb{P}_{\sigma}^{4} \mid \xi_{0}: \xi_{1}=\sigma_{30}: \sigma_{31}\right\}$, hence $P$ is the blowing-up of $\mathbb{P}_{\sigma}^{4}$ with center $\left\{\sigma_{30}=\sigma_{31}=0\right.$ in $\left.\mathbb{P}_{\sigma}^{4}\right\}$. The exceptional divisor $E$ is $\left\{\sigma_{30}=\sigma_{31}=0\right.$ in $\left.P\right\} \in$ $\left|H_{\sigma}-H_{\xi}\right|$, thus $M \in\left|3 H_{\sigma}-E\right|$ and $M$ is the strict transform of a smooth hypercubic in $\mathbb{P}_{\sigma}^{4}$.

(3.22) Suppose that $d=10$. We have $e=6, b=-2$, and $M \in\left|2 H-2 H_{\xi}\right|$. Since $e_{0} \geq 1$ by $(3.15), \mathcal{E} \simeq \mathcal{O}(1,1,1,3), \mathcal{O}(1,1,2,2), \mathcal{O}(1,1,1,1,2)$, or $\mathcal{O}(1,1,1,1,1,1)$.

(3.22.1) When $\mathcal{E} \simeq \mathcal{O}(1,1,1,1,1,1)$, we have $P \simeq \mathbb{P}_{\xi}^{1} \times \mathbb{P}_{\sigma}^{5}, H=H_{\xi}+H_{\sigma}, M \in$ $\left|2 H_{\sigma}\right|$,and $L=\left[H_{\xi}+H_{\sigma}\right]_{M}$. Hence $M \simeq \mathbb{P}_{\xi}^{1} \times Q$, where $Q$ is a smooth hyperquadric in $\mathbb{P}_{\sigma}^{5}$.

(3.22.2) When $\mathcal{E} \simeq \mathcal{O}(1,1,1,1,2)$, by the argument in $[\mathrm{F} 7 ;(3.28)]$, we have $M$ is the blowing-up of a hyperquadric in $\mathbb{P}_{\sigma}^{5}$ and its center is a smooth quadric surface.

(3.22.3) When $\mathcal{E} \simeq \mathcal{O}(1,1,2,2)$, we have $P \simeq\left\{\left(\xi_{0}: \xi_{1}\right) \times\left(\sigma_{0}: \sigma_{1}: \sigma_{20}: \sigma_{21}:\right.\right.$ $\left.\left.\sigma_{30}: \sigma_{31}\right) \in \mathbb{P}_{\xi}^{1} \times \mathbb{P}_{\sigma}^{5} \mid \xi_{0}: \xi_{1}=\sigma_{20}: \sigma_{21}=\sigma_{30}: \sigma_{31}\right\}, H=H_{\xi}+H_{\sigma}, M \in\left|2 H_{\sigma}\right|$, and $L=\left[H_{\xi}+H_{\sigma}\right]_{M}$. Since $\mathcal{E}$ is ample, $H$ is ample and then $L$ is ample for any general member $M$ of $\left|2 H_{\sigma}\right|$. Because of $(3.18), M$ is embedded in $\mathbb{P}^{9}$ as a manifold of degree nine by the morphism defined by $|L|$. On the other hand, the restriction of the projection $\mu: P \rightarrow \mathbb{P}_{\sigma}^{5}$ to $M$ is the morphism defined by $\left|L-H_{\xi}\right|$, and $M$ is birationally mapped onto $\mu(M)$. We have $10=L^{3}=3\left[H_{\xi}\right]_{M}\left[H_{\sigma}\right]_{M}^{2}+\left[H_{\sigma}\right]_{M}^{3}$ and $\left[H_{\xi}\right]_{M}\left[H_{\sigma}\right]_{M}^{2}=2$ since $M \rightarrow \mathbb{P}_{\xi}^{1}$ is a hyperquadric fibration. Thus the degree of $\mu(M)$ is four. Furthermore,since $\mu(P)=\left\{\sigma_{20} \sigma_{31}-\sigma_{30} \sigma_{21}=0\right.$ in $\left.\mathbb{P}_{\sigma}^{5}\right\}$ and $M \in\left|2 H_{\sigma}\right|, \mu(M)$ is a complete intersection of two hyperquadrics in $\mathbb{P}_{\sigma}^{5}$. Even when $\mathcal{E} \simeq \mathcal{O}(1,1,1,3)$, we have the same result as above.

(3.23) Suppose that $d=11$. We have $e=7, b=-3$, and $M \in\left|2 H-3 H_{\xi}\right|$. Since $e_{0} \geq 1$ by (3.15), and since $n=3$ by $(3.5), \mathcal{E} \simeq \mathcal{O}(1,1,1,4), \mathcal{O}(1,1,2,3)$ or $\mathcal{O}(1,2,2,2)$.

(3.23.1) When $\mathcal{E} \simeq \mathcal{O}(1,1,1,4)$,we claim that $(M, L)$ does not exist. Assume that $(M, L)$ exists. A natural surjection $\mathcal{E} \rightarrow \mathcal{O}(1,1,1)$ gives a prime divisor $W:=$ $\mathbb{P}(\mathcal{O}(1,1,1))$ on $P$. Since $W \simeq \mathbb{P}_{\xi}^{1} \times \mathbb{P}_{\sigma}^{2}, H_{W}=H_{\xi}+H_{\sigma}$, and $W \not \subset M$, we have $[M]_{W}=M \cap W \in\left|2 H_{W}-3 H_{\xi}\right|=\left|2 H_{\sigma}-H_{\xi}\right|$. This is a contradiction,thus we have proved the claim. 
We have $P \simeq\left\{\left(\xi_{0}: \xi_{1}\right) \times\left(\sigma_{0}: \sigma_{1}: \sigma_{20}: \sigma_{21}: \sigma_{30}: \sigma_{31}: \sigma_{32}\right) \in \mathbb{P}_{\xi}^{1} \times \mathbb{P}_{\sigma}^{6} \mid \xi_{0}: \xi_{1}=\right.$ $\left.\sigma_{20}: \sigma_{21}=\sigma_{30}: \sigma_{31}=\sigma_{31}: \sigma_{32}\right\}$ and $H=H_{\sigma}+H_{\xi}$. Assume that there exists a smooth member $M$ of $\left|2 H_{\sigma}-H_{\xi}\right|$. Then there is an exact sequence of normal bundles

$$
0 \rightarrow \mathcal{N}_{B / M} \rightarrow \mathcal{N}_{B / P} \rightarrow\left[\mathcal{N}_{M / P}\right]_{B} \rightarrow 0
$$

, where $B:=\mathrm{Bs}\left|2 H_{\sigma}-H_{\xi}\right|=\left\{\sigma_{20}=\sigma_{21}=\sigma_{30}=\sigma_{31}=\sigma_{32}=0\right.$ in $\left.P\right\} \simeq$ $\mathbb{P}(\mathcal{O}(1,1))$. Since $B$ is the complete intersection of $D_{1}:=\left\{\sigma_{20}=\sigma_{21}=0\right.$ in $\left.P\right\} \simeq$ $\mathbb{P}(\mathcal{O}(1,1,3))$ and $D_{2}:=\left\{\sigma_{30}=\sigma_{31}=\sigma_{32}=0\right.$ in $\left.P\right\} \simeq \mathbb{P}(\mathcal{O}(1,1,2))$, we have $\mathcal{N}_{B / P} \simeq\left[\mathcal{N}_{D_{1} / P}\right]_{B} \oplus\left[\mathcal{N}_{D_{2} / P}\right]_{B} \simeq\left[H_{\sigma}-H_{\xi}\right]_{B} \oplus\left[H_{\sigma}-2 H_{\xi}\right]_{B}$. Also we have $\mathcal{N}_{M / P} \simeq$ $\left[2 H_{\sigma}-H_{\xi}\right]_{B}$. Then the morphism $\varphi:\left[H_{\sigma}-H_{\xi}\right]_{B} \oplus\left[H_{\sigma}-2 H_{\xi}\right]_{B} \rightarrow\left[2 H_{\sigma}-H_{\xi}\right]_{B}$ corresponding to $\mathcal{N}_{B / P} \rightarrow\left[\mathcal{N}_{M / P}\right]_{B}$ is given by some $\varphi_{1} \in H^{0}\left(B,\left[H_{\sigma}\right]_{B}\right)$ and $\varphi_{2} \in H^{0}\left(B,\left[H_{\sigma}+H_{\xi}\right]_{B}\right)$. Since $\left[H_{\sigma}\right]_{B}\left[H_{\sigma}+H_{\xi}\right]_{B}=1, \varphi_{1}$ and $\varphi_{2}$ have a common zero point, at which $\varphi$ is not surjective. This yields a contradiction and $(M, L)$ does not exist.

(3.23.3) When $\mathcal{E} \simeq \mathcal{O}(1,2,2,2)$,we can show the existence of $(M, L)$. We have $P \simeq\left\{\left(\xi_{0}: \xi_{1}\right) \times\left(\sigma_{0}: \sigma_{10}: \sigma_{11}: \sigma_{20}: \sigma_{21}: \sigma_{30}: \sigma_{31}\right) \in \mathbb{P}_{\xi}^{1} \times \mathbb{P}_{\sigma}^{6} \mid \xi_{0}: \xi_{1}=\sigma_{10}:\right.$ $\left.\sigma_{11}=\sigma_{20}: \sigma_{21}=\sigma_{30}: \sigma_{31}\right\}$ and $H=H_{\sigma}+H_{\xi}$. Putting $U_{i}=\left\{\xi_{i} \neq 0\right.$ in $\left.P\right\}$ and $V_{j}=\left\{\sigma_{j} \neq 0\right.$ in $\left.P\right\}$, we take a rational section $s_{1}:=\left\{\left(U_{i} \cap V_{j}, \frac{\sigma_{0}^{2}}{\xi_{0}} \cdot \frac{\xi_{i}}{\sigma_{j}^{2}}\right)\right\}_{i, j}$ of $2 H_{\sigma}-H_{\xi}$. Note that $h^{0}\left(P, 2 H-3 H_{\xi}\right)=h^{0}\left(\mathbb{P}_{\xi}^{1}, S^{2}(\mathcal{E}) \otimes\left[-3 H_{\xi}\right]\right)=15$. Let $f_{1}, \ldots, f_{15}$ be rational functions on $P$ such that

$$
\begin{aligned}
f_{1} & =\frac{\xi_{0}}{\sigma_{0}^{2}} \cdot \frac{\sigma_{0} \sigma_{10}}{\xi_{0}}=\frac{\xi_{0}}{\sigma_{0}^{2}} \cdot \frac{\sigma_{0} \sigma_{11}}{\xi_{1}}, \quad f_{2}=\frac{\xi_{0}}{\sigma_{0}^{2}} \cdot \frac{\sigma_{0} \sigma_{20}}{\xi_{0}}=\frac{\xi_{0}}{\sigma_{0}^{2}} \cdot \frac{\sigma_{0} \sigma_{21}}{\xi_{1}}, \\
f_{3} & =\frac{\xi_{0}}{\sigma_{0}^{2}} \cdot \frac{\sigma_{0} \sigma_{30}}{\xi_{0}}=\frac{\xi_{0}}{\sigma_{0}^{2}} \cdot \frac{\sigma_{0} \sigma_{31}}{\xi_{1}}, \quad f_{4}=\frac{\xi_{0}}{\sigma_{0}^{2}} \cdot \frac{\sigma_{10}^{2}}{\xi_{0}}=\frac{\xi_{0}}{\sigma_{0}^{2}} \cdot \frac{\sigma_{10} \sigma_{11}}{\xi_{1}}, \\
f_{5} & =\frac{\xi_{0}}{\sigma_{0}^{2}} \cdot \frac{\sigma_{10} \sigma_{11}}{\xi_{0}}=\frac{\xi_{0}}{\sigma_{0}^{2}} \cdot \frac{\sigma_{11}^{2}}{\xi_{1}}, \quad f_{6}=\frac{\xi_{0}}{\sigma_{0}^{2}} \cdot \frac{\sigma_{10} \sigma_{20}}{\xi_{0}}=\frac{\xi_{0}}{\sigma_{0}^{2}} \cdot \frac{\sigma_{10} \sigma_{21}}{\xi_{1}}, \\
f_{7} & =\frac{\xi_{0}}{\sigma_{0}^{2}} \cdot \frac{\sigma_{10} \sigma_{21}}{\xi_{0}}=\frac{\xi_{0}}{\sigma_{0}^{2}} \cdot \frac{\sigma_{11} \sigma_{21}}{\xi_{1}}, \quad f_{8}=\frac{\xi_{0}}{\sigma_{0}^{2}} \cdot \frac{\sigma_{10} \sigma_{30}}{\xi_{0}}=\frac{\xi_{0}}{\sigma_{0}^{2}} \cdot \frac{\sigma_{10} \sigma_{31}}{\xi_{1}}, \\
f_{9} & =\frac{\xi_{0}}{\sigma_{0}^{2}} \cdot \frac{\sigma_{10} \sigma_{31}}{\xi_{0}}=\frac{\xi_{0}}{\sigma_{0}^{2}} \cdot \frac{\sigma_{11} \sigma_{31}}{\xi_{1}}, f_{10}=\frac{\xi_{0}}{\sigma_{0}^{2}} \cdot \frac{\sigma_{20}^{2}}{\xi_{0}}=\frac{\xi_{0}}{\sigma_{0}^{2}} \cdot \frac{\sigma_{20} \sigma_{21}}{\xi_{1}}, \\
f_{13} & =\frac{\xi_{0}}{\sigma_{0}^{2}} \cdot \frac{\sigma_{20} \sigma_{21}}{\xi_{0}}=\frac{\sigma_{0} \sigma_{31}}{\xi_{0}}=\frac{\xi_{21}^{2}}{\xi_{0}^{2}}, \quad f_{12}=\frac{\xi_{0}}{\sigma_{0}^{2}} \cdot \frac{\sigma_{20} \sigma_{30}}{\xi_{0}}=\frac{\xi_{01}}{\xi_{0}} \cdot \frac{\sigma_{20} \sigma_{31}}{\xi_{0}}, f_{14}=\frac{\xi_{0}}{\xi_{0}^{2}} \cdot \frac{\sigma_{30}^{2}}{\xi_{0}}=\frac{\xi_{0}}{\sigma_{0}^{2}} \cdot \frac{\sigma_{30} \sigma_{31}}{\xi_{1}}, \\
f_{15} & =\frac{\xi_{0}}{\sigma_{0}^{2}} \cdot \frac{\sigma_{30} \sigma_{31}}{\xi_{0}}=\frac{\xi_{0}}{\sigma_{0}^{2}} \cdot \frac{\sigma_{31}^{2}}{\xi_{1}} .
\end{aligned}
$$

Then $\mathbb{C}\left\langle f_{1}, \ldots, f_{15}\right\rangle$, the vector space spanned by $f_{1}, \ldots, f_{15}$ over $\mathbb{C}$, is isomorphic to $H^{0}\left(P, 2 H_{\sigma}-H_{\xi}\right)$ by mapping each $f_{i}$ to $f_{i} \cdot s_{1}$. Thus we can describe

$$
\left|2 H_{\sigma}-H_{\xi}\right|=\left\{\operatorname{div}\left(f \cdot s_{1}\right) \mid f \in \mathbb{C}\left\langle f_{1}, \ldots, f_{15}\right\rangle-0\right\}
$$

, where $\operatorname{div}\left(f \cdot s_{1}\right)$ is an effective divisor defined by a regular section $f \cdot s_{1}$ of $2 H_{\sigma}-H_{\xi}$. 
if we take $f=\sum_{i=1}^{15} c_{i} f_{i} \in \mathbb{C}\left\langle f_{1}, \ldots, f_{15}\right\rangle$ with $\left(c_{1}, c_{2}, c_{3}\right) \neq(0,0,0), \operatorname{div}\left(f \cdot s_{1}\right)$ is nonsingular along $\mathrm{Bs}\left|2 H_{\sigma}-H_{\xi}\right|$. Thus any general member $M$ of $\left|2 H_{\sigma}-H_{\xi}\right|$ is smooth by Bertini's theorem. For such $M, L:=H_{M}$ is ample since $\mathcal{E}$ is ample, hence $(M, L)$ is a polarized manifold as desired. Furthermore,similarly as in (3.16.3), $\left|L-H_{\xi}\right|$ makes $M$ a desingularization of a variety of degree five in $\mathbb{P}_{\sigma}^{6}$.

(3.24) Suppose that $d=12$. We have $e=8, b=-4$, and $M \in\left|2 H-4 H_{\xi}\right|$. Since $e_{0} \geq 1$ by $(3.15)$, and since $n=3$ by $(3.5), \mathcal{E} \simeq \mathcal{O}(1,1,1,5), \mathcal{O}(1,1,2,4), \mathcal{O}(1,1,3,3), \mathcal{O}(1,2,2,3)$,or $\mathcal{O}(2,2,2,2)$.

(3.24.1) When $\mathcal{E} \simeq \mathcal{O}(2,2,2,2)$, we have $P \simeq \mathbb{P}_{\xi}^{1} \times \mathbb{P}_{\sigma}^{3}, H=H_{\sigma}+2 H_{\xi}, M \in\left|2 H_{\sigma}\right|$, and $L=\left[H_{\sigma}+H_{\xi}\right]_{M}$. Hence $M \simeq \mathbb{P}_{\xi}^{1} \times Q$, where $Q$ is a smooth quadric surface in $\mathbb{P}_{\sigma}^{3}$. Since $Q \simeq \mathbb{P}_{\mu}^{1} \times \mathbb{P}_{\lambda}^{1}$, we have $M \simeq \mathbb{P}_{\xi}^{1} \times \mathbb{P}_{\mu}^{1} \times \mathbb{P}_{\lambda}^{1}$ and $L=2 H_{\xi}+H_{\mu}+H_{\lambda}$.

(3.24.2) When $\mathcal{E} \simeq \mathcal{O}(1,1,1,5),(M, L)$ does not exist by the argument in (3.23.1).

(3.24.3) Even when $\mathcal{E} \simeq \mathcal{O}(1,1,2,4)$, we can show that $(M, L)$ does not exist similarly as in (3.23.2).

(3.24.4) When $\mathcal{E} \simeq \mathcal{O}(1,2,2,3)$, we can show the existence of $(M, L)$ similarly as in (3.23.3). In fact,we have $P \simeq\left\{\left(\xi_{0}: \xi_{1}\right) \times\left(\sigma_{0}: \sigma_{10}: \sigma_{11}: \sigma_{20}: \sigma_{21}: \sigma_{30}:\right.\right.$ $\left.\left.\sigma_{31}: \sigma_{32}\right) \in \mathbb{P}_{\xi}^{1} \times \mathbb{P}_{\sigma}^{7} \mid \xi_{0}: \xi_{1}=\sigma_{10}: \sigma_{11}=\sigma_{20}: \sigma_{21}=\sigma_{30}: \sigma_{31}=\sigma_{31}: \sigma_{32}\right\}$, $H=H_{\sigma}+H_{\xi}$, and $h^{0}\left(P, 2 H-4 H_{\xi}\right)=h^{0}\left(\mathbb{P}_{\xi}^{1}, S^{2}(\mathcal{E}) \otimes\left[-4 H_{\xi}\right]\right)=11$. We take a rational section $s_{2}:=\left\{\left(U_{i} \cap V_{j}, \frac{\sigma_{0}^{2}}{\xi_{0}^{2}} \cdot \frac{\xi_{i}^{2}}{\sigma_{j}^{2}}\right)\right\}_{i, j}$ of $2 H_{\sigma}-2 H_{\xi}$, where $U_{i}$ and $V_{j}$ are the same as in (3.23.3). Let $f_{1}, \ldots, f_{11}$ be rational functions on $P$ such that

$$
\begin{aligned}
& f_{1}=\frac{\xi_{0}^{2}}{\sigma_{0}^{2}} \cdot \frac{\sigma_{0} \sigma_{30}}{\xi_{0}^{2}}, \quad f_{2}=\frac{\xi_{0}^{2}}{\sigma_{0}^{2}} \cdot \frac{\sigma_{10}^{2}}{\xi_{0}^{2}}, \quad f_{3}=\frac{\xi_{0}^{2}}{\sigma_{0}^{2}} \cdot \frac{\sigma_{10} \sigma_{20}}{\xi_{0}^{2}}, f_{4}=\frac{\xi_{0}^{2}}{\sigma_{0}^{2}} \cdot \frac{\sigma_{10} \sigma_{30}}{\xi_{0}^{2}}, \\
& f_{5}=\frac{\xi_{0}^{2}}{\sigma_{0}^{2}} \cdot \frac{\sigma_{10} \sigma_{31}}{\xi_{0}^{2}}, \quad f_{6}=\frac{\xi_{0}^{2}}{\sigma_{0}^{2}} \cdot \frac{\sigma_{20}^{2}}{\xi_{0}^{2}}, \quad f_{7}=\frac{\xi_{0}^{2}}{\sigma_{0}^{2}} \cdot \frac{\sigma_{20} \sigma_{30}}{\xi_{0}^{2}}, f_{8}=\frac{\xi_{0}^{2}}{\sigma_{0}^{2}} \cdot \frac{\sigma_{20} \sigma_{31}}{\xi_{0}^{2}}, \\
& f_{9}=\frac{\xi_{0}^{2}}{\sigma_{0}^{2}} \cdot \frac{\sigma_{30}^{2}}{\xi_{0}^{2}}, \quad f_{10}=\frac{\xi_{0}^{2}}{\sigma_{0}^{2}} \cdot \frac{\sigma_{30} \sigma_{31}}{\xi_{0}^{2}}, f_{11}=\frac{\xi_{0}^{2}}{\sigma_{0}^{2}} \cdot \frac{\sigma_{30} \sigma_{32}}{\xi_{0}^{2}} .
\end{aligned}
$$

Then $H^{0}\left(P, 2 H_{\sigma}-2 H_{\xi}\right) \simeq \mathbb{C}\left\langle f_{1}, \ldots, f_{11}\right\rangle$ and $\mathrm{Bs}\left|2 H_{\sigma}-2 H_{\xi}\right|=\mathbb{P}_{\xi}^{1} \times\{(1: 0:$ $\cdots: 0)\}$. For any $f=\sum_{i=1}^{11} c_{i} f_{i}$ with $c_{1} \neq 0, \operatorname{div}\left(f \cdot s_{2}\right)$ is nonsingular along $\mathrm{Bs}\left|2 H_{\sigma}-2 H_{\xi}\right|$, thus any general member $M$ of $\left|2 H_{\sigma}-2 H_{\xi}\right|$ is smooth. Putting $L=H_{M}$, we obtain a polarized manifold $(M, L)$ as desired. In this case, $\left|L-H_{\xi}\right|$ makes $M$ a desingularization of a variety of degree six in $\mathbb{P}^{7}$.

(3.24.5) Even when $\mathcal{E} \simeq \mathcal{O}(1,1,3,3)$, we can show the existence of $(M, L)$ similarly. We have $P \simeq\left\{\left(\xi_{0}: \xi_{1}\right) \times\left(\begin{array}{llllllll}\sigma_{0}: \sigma_{1}: \sigma_{20}: \sigma_{21}: \sigma_{22}: \sigma_{30}: \sigma_{31}:\end{array}\right.\right.$ $\left.\left.\sigma_{32}\right) \in \mathbb{P}_{\xi}^{1} \times \mathbb{P}_{\sigma}^{7} \mid \xi_{0}: \xi_{1}=\sigma_{20}: \sigma_{21}=\sigma_{21}: \sigma_{22}=\sigma_{30}: \sigma_{31}=\sigma_{31}: \sigma_{32}\right\}$ and $H^{0}\left(P, 2 H_{\sigma}-2 H_{\xi}\right) \simeq \mathbb{C}\left\langle f_{1}, \ldots, f_{13}\right\rangle$, where

$$
\begin{aligned}
f_{1} & =\frac{\xi_{0}^{2}}{\sigma_{0}^{2}} \cdot \frac{\sigma_{0} \sigma_{20}}{\xi_{0}^{2}}, \quad f_{2}=\frac{\xi_{0}^{2}}{\sigma_{0}^{2}} \cdot \frac{\sigma_{0} \sigma_{30}}{\xi_{0}^{2}}, \quad f_{3}=\frac{\xi_{0}^{2}}{\sigma_{0}^{2}} \cdot \frac{\sigma_{1} \sigma_{20}}{\xi_{0}^{2}}, \quad f_{4}=\frac{\xi_{0}^{2}}{\sigma_{0}^{2}} \cdot \frac{\sigma_{1} \sigma_{30}}{\xi_{0}^{2}} \\
f_{5} & =\frac{\xi_{0}^{2}}{\sigma_{0}^{2}} \cdot \frac{\sigma_{20}^{2}}{\xi_{0}^{2}}, \quad f_{6}=\frac{\xi_{0}^{2}}{\sigma_{0}^{2}} \cdot \frac{\sigma_{20} \sigma_{21}}{\xi_{0}^{2}}, \quad f_{7}=\frac{\xi_{0}^{2}}{\sigma_{0}^{2}} \cdot \frac{\sigma_{21}^{2}}{\xi_{0}^{2}}, \quad f_{8}=\frac{\xi_{0}^{2}}{\sigma_{0}^{2}} \cdot \frac{\sigma_{20} \sigma_{30}}{\xi_{0}^{2}} \\
f_{9} & =\frac{\xi_{0}^{2}}{\sigma_{0}^{2}} \cdot \frac{\sigma_{20} \sigma_{31}}{\xi_{0}^{2}}, f_{10}=\frac{\xi_{0}^{2}}{\sigma_{0}^{2}} \cdot \frac{\sigma_{21} \sigma_{31}}{\xi_{0}^{2}}, f_{11}=\frac{\xi_{0}^{2}}{\sigma_{0}^{2}} \cdot \frac{\sigma_{30}^{2}}{\xi_{0}^{2}}, \quad f_{12}=\frac{\xi_{0}^{2}}{\sigma_{0}^{2}} \cdot \frac{\sigma_{30} \sigma_{31}}{\xi_{0}^{2}} \\
f_{13} & =\frac{\xi_{0}^{2}}{2} \cdot \frac{\sigma_{31}^{2}}{\omega} .
\end{aligned}
$$


Since Bs $\left|2 H_{\sigma}-2 H_{\xi}\right|=\left\{\sigma_{20}=\sigma_{21}=\cdots=\sigma_{32}=0\right.$ in $\left.P\right\}$, if we take $f=\sum_{i=1}^{13} c_{i} f_{i}$ with $c_{1} c_{4}-c_{2} c_{3} \neq 0, \operatorname{div}\left(f \cdot s_{2}\right)$ is nonsingular along $\mathrm{Bs}\left|2 H_{\sigma}-2 H_{\xi}\right|$. Thus any general member $M$ of $\left|2 H_{\sigma}-2 H_{\xi}\right|$ is smooth. Putting $L=H_{M}$, we obtain a polarized manifold $(M, L)$ as desired, and $\left|L-H_{\xi}\right|$ makes $M$ a desingularization of a variety of degree six in $\mathbb{P}^{7}$.

Summarizing the results in $\S 3$,we obtain the following.

(3.25)Theorem. Let $(M, L)$ be a polarized manifold of the type (1.5.3). Then $g(C)$, the genus of $C$, is 0 or $1, \mathcal{E}:=\Phi_{*} \mathcal{O}_{M}[L]$ is a locally free sheaf on $C, M \in$ $\left|2 H(\mathcal{E})+B_{\mathbb{P}(\mathcal{E})}\right|$ for some line bundle $B$ on $C$, and $L=[H(\mathcal{E})]_{M}$. Putting $d=$ $L^{n}, e=c_{1}(\mathcal{E})$, and $b=\operatorname{deg} B$, we have the following results.

When $g(C)=1$, we have $1 \leq d \leq 6, e=d-2, b=4-d$, and

(i) if $d=1$ or 2 , then $\mathcal{E}$ is not ample;

(ii) if $d=3$ or 4 , then $\mathcal{E}$ is ample as long as it is indecomposable;

(iii) if $d=5$ or 6 , then $\mathcal{E}$ is ample.

When $g(C)=0$, we have $C \simeq \mathbb{P}_{\xi}^{1}, 1 \leq d \leq 12$, e $=d-4, b=8-d, M \in$ $\left|2 H(\mathcal{E})+b H_{\xi}\right|$, and their lists are in the table below. 


\begin{tabular}{|c|c|c|}
\hline$d$ & $\mathcal{E}$ & $(M, L)$ \\
\hline \multirow[t]{6}{*}{1} & $\mathcal{O}(-3,0,0,0)$ & The existence is uncertain. \\
\hline & $\mathcal{O}(-3,0,0,0,0)$ & $\prime \prime$ \\
\hline & $\mathcal{O}(-2,-1,0,0)$ & $\prime \prime$ \\
\hline & $\mathcal{O}(-2,-1,0,0,0)$ & $\prime \prime$ \\
\hline & $\mathcal{O}(-1,-1,-1,0)$ & $\prime \prime$ \\
\hline & $\mathcal{O}(-1,-1,-1,0,0)$ & $\prime \prime$ \\
\hline \multirow[t]{4}{*}{2} & $\mathcal{O}(-2,0,0,0)$ & 11 \\
\hline & $\mathcal{O}(-2,0,0,0,0)$ & $\prime \prime$ \\
\hline & $\mathcal{O}(-1,-1,0,0)$ & $\prime \prime$ \\
\hline & $\mathcal{O}(-1,-1,0,0,0)$ & $\prime \prime$ \\
\hline \multirow[t]{6}{*}{3} & $\mathcal{O}(-2,0,0,1)$ & 11 \\
\hline & $\mathcal{O}(-2,0,0,0,1)$ & $\prime \prime$ \\
\hline & $\mathcal{O}(-1,-1,0,1)$ & $\prime \prime$ \\
\hline & $\mathcal{O}(-1,-1,0,0,1)$ & $\prime \prime$ \\
\hline & $\mathcal{O}(-1,0,0,0)$ & $\prime \prime$ \\
\hline & $\mathcal{O}(-1,0,0,0,0)$ & $\prime \prime$ \\
\hline \multirow[t]{4}{*}{4} & $\mathcal{O}(-1,0,0,1)$ & "1 \\
\hline & $\mathcal{O}(-1,0,0,0,1)$ & 11 \\
\hline & $\mathcal{O}(0,0,0,0)$ & $|L|$ makes $M$ a quadruple covering of $\mathbb{P}^{3}$. \\
\hline & $\mathcal{O}(0,0,0,0,0)$ & $|L|$ makes $M$ a quadruple covering of $\mathbb{P}^{4}$. \\
\hline \multirow[t]{5}{*}{5} & $\mathcal{O}(-1,0,0,2)$ & $\mathrm{Bs}|L|$ is a point. \\
\hline & $\mathcal{O}(-1,0,1,1)$ & 11 \\
\hline & $\mathcal{O}(-1,0,0,1,1)$ & " \\
\hline & $\mathcal{O}(0,0,0,1)$ & $|L|$ makes $M$ the normalization of a hypersurface of degree five in $\mathbb{P}^{4}$. \\
\hline & $\mathcal{O}(0,0,0,0,1)$ & $|L|$ makes $M$ the normalization of a hypersurface of degree five in $\mathbb{P}^{5}$. \\
\hline \multirow[t]{5}{*}{6} & $\mathcal{O}(-1,1,1,1)$ & $M$ is a double covering of $\mathbb{P}_{\xi}^{1} \times \mathbb{P}_{\sigma}^{2}$ with branch locus being \\
\hline & & a smooth divisor of bidegree $(4,2) . L=\left[H_{\xi}+H_{\sigma}\right]_{M}$ \\
\hline & $\mathcal{O}(0,0,1,1)$ & Exist. \\
\hline & $\mathcal{O}(0,0,0,1,1)$ & "1 \\
\hline & $\mathcal{O}(0,0,0,2)$ & $\prime \prime$ \\
\hline \multirow[t]{3}{*}{7} & $\mathcal{O}(0,0,1,2)$ & '1" \\
\hline & $\mathcal{O}(0,1,1,1)$ & $\prime \prime$ \\
\hline & $\mathcal{O}(0,0,1,1,1)$ & 11 \\
\hline \multirow[t]{3}{*}{8} & $\mathcal{O}(0,1,1,1,1)$ & $\begin{array}{c}M \text { is a double covering of } \mathbb{P}_{\xi}^{1} \times \mathbb{P}_{\sigma}^{3} \text { with branch locus being } \\
\text { a smooth divisor of bidegree }(2,2) . L=\left[H_{\xi}+H_{\sigma}\right]_{M} .\end{array}$ \\
\hline & $\mathcal{O}(0,1,1,2)$ & $\begin{array}{c}M \text { is a double covering of a divisor of bidegree }(1,1) \\
\qquad \text { on } \mathbb{P}_{\xi}^{1} \times \mathbb{P}_{\sigma}^{3} \cdot L=\left[H_{\xi}+H_{\sigma}\right]_{M}\end{array}$ \\
\hline & $\mathcal{O}(1,1,1,1)$ & $M$ is a smooth divisor of bidegree $(2,2)$ on $\mathbb{P}_{\xi}^{1} \times \mathbb{P}_{\sigma}^{3} . L=\left[H_{\xi}+H_{\sigma}\right]_{M}$. \\
\hline 9 & $\mathcal{O}(1,1,1,1,1)$ & $\begin{array}{l}M \text { is the blowing-up of } \mathbb{P}_{\sigma}^{4} \text { with center being a complete } \\
\text { intersection of two hyperquadrics. } L=\left[H_{\xi}+H_{\sigma}\right]_{M} .\end{array}$ \\
\hline & $\mathcal{O}(1,1,1,2)$ & $\begin{array}{l}M \text { is the strict transform of a smooth hyperqubic in } \mathbb{P}_{\sigma}^{4} \text { by the } \\
\text { blowing-up of } \mathbb{P}_{\sigma}^{4} \text { with center being a } \mathbb{P}^{2} . L=\left[H_{\xi}+H_{\sigma}\right]_{M} .\end{array}$ \\
\hline 10 & $\begin{array}{l}\mathcal{O}(1,1,1,1,1,1) \\
\mathcal{O}(1,1,1,1,2)\end{array}$ & $\begin{array}{c}M \simeq \mathbb{P}_{\xi}^{1} \times Q, \text { where } Q \text { is a smooth hyperquadric in } \mathbb{P}_{\sigma}^{5} \cdot L=\left[H_{\xi}+H_{\sigma}\right]_{M} \cdot \\
M \text { is the blowing-up of a hyperquadric in } \mathbb{P}_{\sigma}^{5} \text { with center } \\
\text { being a smooth auadric surface. } L=\left[H_{c}+H_{\sigma}\right]_{M .}\end{array}$ \\
\hline
\end{tabular}




\begin{tabular}{|c|c|c|}
\hline$d$ & $\mathcal{E}$ & $(M, L)$ \\
\hline 10 & $\mathcal{O}(1,1,2,2)$ & $M$ is a desingularization of a complete intersection of \\
& & two hyperquadrics in $\mathbb{P}_{\sigma}^{5} \cdot L=\left[H_{\xi}+H_{\sigma}\right]_{M}$. \\
& $\mathcal{O}(1,1,1,3)$ & " \\
\hline 11 & $\mathcal{O}(1,2,2,2)$ & $\left|L-H_{\xi}\right|$ makes $M$ a desingularization of a three- \\
& & dimensional variety of degree five in $\mathbb{P}^{6}$. \\
\hline 12 & $\mathcal{O}(1,1,3,3)$ & $\left|L-H_{\xi}\right|$ makes $M$ a desingularization of a three- \\
& & dimensional variety of degree six in $\mathbb{P}^{7}$. \\
& $\mathcal{O}(1,2,2,3)$ & ' \\
& $\mathcal{O}(2,2,2,2)$ & $M \simeq \mathbb{P}_{\xi}^{1} \times \mathbb{P}_{\mu}^{1} \times \mathbb{P}_{\lambda}^{1}$ and $L=2 H_{\xi}+H_{\mu}+H_{\lambda .}$. \\
\hline
\end{tabular}

\section{$\S 4$ The case of a Veronese fibration over a curve.}

In this section we study the case (2) of the theorem (1.5), using the argument in $[\mathrm{F} ;(\mathrm{II} .13 .10)]$.

(4.1) Put $H=K+2 L$, then $\mathcal{E}:=\Phi_{*} \mathcal{O}_{M}[H]$ is a locally free sheaf of rank three on $C$ and $(M, H)$ is the scroll of $\mathcal{E}$. We have $L=2 H+\Phi^{*} B$ for some $B \in \operatorname{Pic}(C)$. Similarly as before,we put $d=L^{3}, e=c_{1}(\mathcal{E}), b=\operatorname{deg} B$ and denote by $g(C)$ the genus of $C$. Then $e \geq 0, e+b=1$, and $d=8 e+12 b$. By the canonical bundle formula,we obtain that $K^{C}+\operatorname{det} \mathcal{E}+2 B=0$, hence $2 g(C)-2+e+2 b=0$. From these results, $(e, d)=(0,12)$ or $(2,4)$.

(4.2) When $(e, d)=(0,12)$, we have $b=1$ and $g(C)=0$, hence $C \simeq \mathbb{P}^{1}, B=$ $\mathcal{O}(1)$, and $\mathcal{E} \simeq \mathcal{O}\left(e_{1}\right) \oplus \mathcal{O}\left(e_{2}\right) \oplus \mathcal{O}\left(e_{3}\right)$ for $e_{1}, e_{2}, e_{3} \in \mathbb{Z}$. For each $1 \leq i \leq 3$,a natural surjection $\mathcal{E} \rightarrow \mathcal{O}\left(e_{i}\right)$ gives a section $Z_{i}$ of $\Phi$ and $H_{Z_{i}}=\mathcal{O}\left(e_{i}\right)$. Since $e_{1}+e_{2}+e_{3}=e=0$ and $L_{Z_{i}}=\mathcal{O}\left(2 e_{i}+1\right)$ is ample, we have $e_{1}=e_{2}=e_{3}=0$ and $\mathcal{E} \simeq \mathcal{O}_{C}^{\oplus 3}$, thus $M \simeq \mathbb{P}_{\xi}^{1} \times \mathbb{P}_{\sigma}^{2}$ and $L=H_{\xi}+2 H_{\sigma}$. We note that this $(M, L)$ is already obtained in (2.9.3).

(4.3) When $(e, d)=(2,4)$, we have $b=-1$ and $g(C)=1$. Hence $C$ is an elliptic curve and $\operatorname{det} \mathcal{E}+2 B=0$ since $K^{C}=\mathcal{O}_{C}$. Let $Q$ be any quotient bundle of $\mathcal{E}$. If $\operatorname{rank} Q=1$, then $Z:=\mathbb{P}_{C}(Q)$ is a section of $\Phi$ and $H Z=c_{1}(Q)$. Then $c_{1}(Q) \geq 1$ since $0<L Z=2 c_{1}(Q)-1$. If rank $Q=2$, then $D:=\mathbb{P}_{C}(Q) \in\left|H-\Phi^{*} \mathcal{F}\right|$, where $\mathcal{F}$ is the kernel of $\mathcal{E} \rightarrow Q$. Since $0<L^{2} D=4\left(1-c_{1}(\mathcal{F})\right)$, we have $c_{1}(Q)=$ $e-c_{1}(\mathcal{F}) \geq 2$. In both cases we have $(\operatorname{rank} Q) \cdot c_{1}(\mathcal{E})<(\operatorname{rank} \mathcal{E}) \cdot c_{1}(Q)$, hence $\mathcal{E}$ is stable. Conversely,let $\mathcal{E}$ be a semistable vector bundle on $C$ with rank $\mathcal{E}=3$ and $c_{1}(\mathcal{E})=2$. We put $M=\mathbb{P}_{C}(\mathcal{E}), H=H(\mathcal{E})$ and let $\Phi: M \rightarrow C$ be the bundle map. By the semistability criterion in $[\mathrm{Mi} ;(3.1)], 3 H-\Phi^{*}(\operatorname{det} \mathcal{E})$ is nef. Since $C$ is an elliptic curve,we can find some $B \in \operatorname{Pic}(C)$ satisfying $\operatorname{det} \mathcal{E}+2 B=0$. Then $3\left(2 H+\Phi^{*} B\right)=2\left(3 H+\Phi^{*}(2 B)\right)-\Phi^{*} B$ is ample. Hence $L:=2 H+\Phi^{*} B$ is ample and $(M, L)$ is a polarized manifold of the type (1.5.2).

(4.4) Summing up,we obtain the following theorem.

Theorem. Let $(M, L)$ be a polarized manifold of the type (1.5.2). We put $d=L^{3}$ and denote by $g(C)$ the genus of $C$. Then $(M, L)$ is one of the following two types.

(I) $g(C)=0$, hence $C \simeq \mathbb{P}_{\xi}^{1} ; d=12, M \simeq \mathbb{P}_{\xi}^{1} \times \mathbb{P}_{\sigma}^{2}$, and $L=H_{\xi}+2 H_{\sigma}$.

(II) $g(C)=1$ and $M \simeq \mathbb{P}_{C}(\mathcal{E})$, where $\mathcal{E}:=\Phi_{*} \mathcal{O}_{M}[K+2 L]$ is a stable vector bundle of rank three on $C$ with $c_{1}(\mathcal{E})=2 ; d=4$ and $L=2 H(\mathcal{E})+\Phi^{*} B$, where $B \in \operatorname{Pic}(C)$ with $\operatorname{det} \mathcal{E}+2 B=0$. 
(5.1) We first study the case (1.5.5). This case is a kind of "general type" and we mainly study $(M, L)$ of small $\Delta$-genus. Since $L$ is ample, $0 \leq(K+(n-2) L) L^{n-1}=$ $2 g-2-L^{n}=4-d$, where $d=L^{n}$. Hence we have $1 \leq d \leq 4$. For any polarized manifold, $\Delta=0$ implies $g=0([\mathrm{~F} 1 ;(1.9)]$ and $[\mathrm{F} 2 ;(4.1)])$. Thus here we have $\Delta \geq 1$. The case $d=1$ is difficult and yet to be studied.

(5.2) Suppose that $d=2$. When $\Delta=1,|L|$ makes $M$ a double covering of $\mathbb{P}^{n}$ with branch locus being a smooth hypersurface of degree eight $([\mathrm{F} 3 ;(2.5)])$. When $\Delta=2, \mathrm{Bs}|L|$ is a finite set $([\mathrm{F} 1 ;(1.9)]$ and $[\mathrm{F} 5 ;(1.17)])$. When $\Delta \geq 3$, we do not have any satisfactory result.

(5.3) Suppose that $d=3$. Then we have $\Delta \neq 1$ by $[\mathrm{F} 1 ;(1.9)]$ and $[\mathrm{F} 2 ;(4.1)]$. When $\Delta=2, \operatorname{dim} \mathrm{Bs}|L| \leq 0([\mathrm{~F} 1 ;(1.9)]$ and $[\mathrm{F} 5 ;(1.14 .5)])$. If $\Delta=2$ and $\mathrm{Bs}|L|=$ $\phi,|L|$ makes $M$ a triple covering of $\mathbb{P}^{n}$. If $\Delta=2$ and $\operatorname{Bs}|L| \neq \phi$, we have the following results in [F9].

a) $\mathrm{Bs}|L|$ consists of one simple point $p$.

b) Let $\pi: M_{1} \rightarrow M$ be the blowing-up at $p$ and let $E$ be the exceptional divisor over $p$. Then $\mathrm{Bs}\left|\pi^{*} L-E\right|=\phi$.

c) $\left|\pi^{*} L-E\right|$ gives a morphism $\rho: M_{1} \rightarrow \mathbb{P}^{n}$ of degree two.Every fiber $X$ of $\rho$ with $\operatorname{dim} X>0$ is an irreducible curve such that $E X=1$.

d) $\rho(E)$ is a hyperplane in $\mathbb{P}^{n}$.

When $\Delta \geq 3$, we do not have any satisfactory result.

(5.4) Suppose that $d=4$. Then we have $(K+(n-2) L) L^{n-1}=0$. Since $K+(n-$ 2) $L$ is nef,by the base point free theorem (cf. [KMM]) and [F5;Appendix],there is a fibration $f: M \rightarrow V$ and an ample line bundle $A$ on $V$ such that $K+(n-2) L=f^{*} A$. Thus we have $K+(n-2) L=0$ since $\left(f^{*} A\right) L^{n-1}=0$. Then $\Delta(M, L)=2$ by $[\mathrm{F} 4 ;(1.11)]$, hence $\operatorname{dim} \mathrm{Bs}|L| \leq 1$ by $[\mathrm{F} 1 ;(1.9)]$. When $\operatorname{dim} \mathrm{Bs}|L|=1, n \leq 4$ by $[\mathrm{F} 5 ;(1.17)]$, and we have the following results by $[\mathrm{F} 5 ;(1.14) \&(2.4)]$.

a) $Y:=\mathrm{Bs}|L|$ is a smooth rational curve.

b) Let $\pi: M^{\prime} \rightarrow M$ be the blowing-up of $Y$ and let $E$ be the exceptional divisor over $Y$. Then $\mathrm{Bs}\left|\pi^{*} L-E\right|=\phi$.

c) Let $W$ be the image of the morphism $M^{\prime} \rightarrow \mathbb{P}^{n+1}$ defined by $\left|\pi^{*} L-E\right|$. Then $\operatorname{dim} W=n-1, \operatorname{deg} W=3$ and $\Delta\left(W, \mathcal{O}_{W}(1)\right)=0$.

d) $E$ is a section of the morphism $\rho: M^{\prime} \rightarrow W$.

e) $\rho$ is flat and every fiber of $\rho$ is an irreducible curve of arithmetic genus one.

f) $M^{\prime}$ is a double covering of a $\mathbb{P}^{1}$-bundle $V:=\mathbb{P}\left(\mathcal{O}_{E} \oplus[-2 E]_{E}\right)$ over $E \simeq W$. The image $S$ of $E$ by the morphism $\beta: M^{\prime} \rightarrow V$ is the unique member of $\left|H_{\zeta}-[-2 E]_{V}\right|$, where $H_{\zeta}$ is the tautological bundle on $V$. The branch locus $B$ of $\beta$ is $B^{*}+S$, where $B^{*}$ is a smooth member of $\left|3 H_{\zeta}\right|$ with $B^{*} \cap S=\phi$.

When $\operatorname{dim} \mathrm{Bs}|L| \leq 0$, we have $\mathrm{Bs}|L|=\phi$ by $[\mathrm{F} 2 ;(4.1)]$. Let $\rho$ be the morphism $M \rightarrow$ $\mathbb{P}^{n+1}$ defined by $|L|$. We put $W=\rho(M)$ and $w=\operatorname{deg} W$,then $4=L^{n}=w \cdot \operatorname{deg} \rho$. Hence $(\operatorname{deg} \rho, w)=(1,4)$ or $(2,2)$. In the former case, $\rho$ is birational and moreover $M \simeq W$ by $[\mathrm{F} ;(10.8 .1)]$. In the latter case, $\rho$ is a double covering of a hyperquadric $W$. Furthermore $W$ turns out to be smooth, and the branch locus of $\rho$ is a smooth hypersurface section and is connected $([\mathrm{F} ;(10.8 .2)])$.

(5.5) Finally we study the case (1) of the theorem (1.5). We use the theory of minimal reduction in $[\mathrm{F} 7 ;(1.9)]$ and $[\mathrm{F} ;(11.11)]$. Clearly $M$ is the blowing-up of another manifold $M_{1}$ at one point and $E$ is the exceptional divisor with $L E=1$. Moreover there is an ample line bundle $L_{1}$ on $M_{1}$ such that $L+E$ is the pullback 
above theory,we obtain a sequence of simple blow-ups

$$
(M, L) \rightarrow\left(M_{1}, L_{1}\right) \rightarrow \cdots \rightarrow\left(M_{r}, L_{r}\right)=\left(M^{\prime}, L^{\prime}\right)
$$

with the following properties:

a) $K+(n-1) L=\left[K^{\prime}+(n-1) L^{\prime}\right]_{M}$ for the canonical bundle $K^{\prime}$ of $M^{\prime}$, hence $K^{\prime}+(n-1) L^{\prime}$ is nef and $g\left(M^{\prime}, L^{\prime}\right)=g(M, L)=3$;

b) $L^{n}=\left(L^{\prime}\right)^{n}-r$;

c) $\left(M^{\prime}, L^{\prime}\right)$ is not of the type (1.5.1) i.e. $\left(M^{\prime}, L^{\prime}\right)$ is minimal.

If $\left(M^{\prime}, L^{\prime}\right)$ is of the type (1.5.3) or (1.5.4), then we can derive a contradiction as in $[$ F7; $(1.9)]$. Thus $\left(M^{\prime}, L^{\prime}\right)$ is of the type $(1.5 .2)$ or $(1.5 .5)$.

(5.6) When $\left(M^{\prime}, L^{\prime}\right)$ is of the type (1.5.2), we can apply the argument in $\S 4$. If $M^{\prime} \simeq \mathbb{P}_{\xi}^{1} \times \mathbb{P}_{\sigma}^{2}$ and $L^{\prime}=H_{\xi}+2 H_{\sigma}$, then we can find a curve $Z$ on $M^{\prime}$ with $L^{\prime} Z=1$. This is a contradiction by $[\mathrm{F} 7 ;(1.9)]$, thus $\left(M^{\prime}, L^{\prime}\right)$ is of the type (4.4.II). Since $4=\left(L^{\prime}\right)^{3}=L^{3}+r$, the number of points at which $M^{\prime}$ is blown up is less than four.

(5.7) When $\left(M^{\prime}, L^{\prime}\right)$ is of the type (1.5.5), $0 \leq\left(K^{\prime}+(n-2) L^{\prime}\right)\left(L^{\prime}\right)^{n-1}=$ $4-\left(L^{\prime}\right)^{n}$ since $K^{\prime}+(n-2) L^{\prime}$ is nef. Then we have $2 \leq L^{n}+r=\left(L^{\prime}\right)^{n} \leq 4$, hence $\left(L^{n}, r,\left(L^{\prime}\right)^{n}\right)=(1,1,2),(1,2,3),(1,3,4),(2,2,4)$,or $(3,1,4)$.

\section{REFERENCES}

[Be] A. Beauville, Surfaces algébriques complexes, Astérisque 54 (1978).

[BeLP] M. Beltrametti,A. Lanteri and M. Palleschi, Algebraic surfaces containing an ample divisor of arithmetic genus two, Arkiv för mat. 25 (1987), 189-210.

[BiLL] A. Biancofiore,A. Lanteri and E. L. Livorni, Ample and spanned vector bundles of sectional genus three, Math. Ann. 291 (1991), 87-101.

[Bo] E. Bombieri, Canonical models of surfaces of general type, Publ. Math. IHES 42 (1973), 171-219.

[E] G. Elencwajg, Les fibrés uniformes de rang 3 sur $\mathbb{P}^{2}(\mathbb{C})$, Math. Ann. 231 (1978), 217-227.

[F] T. Fujita, Classification Theories of Polarized Varieties, London Math. Soc. Lect. Note Series, vol. 155, Cambridge Univ. Press, 1990.

[F1] _ On the structure of polarized varieties with $\Delta$-genera zero, J. Fac. Sci. Univ. of Tokyo 22 (1975), 103-115.

[F2] - Defining equations for certain types of polarized varieties, Complex Analysis and Algebraic Geometry (Baily and Shioda, eds.), Iwanami, 1977, pp. 165-173.

[F3] - On the structure of polarized manifolds with total deficiency one, part I, J. Math. Soc. Japan 32 (1980), 709-725.

[F4] _ On hyperelliptic polarized varieties, Tohoku Math. J. 35 (1983), 1-44.

[F5] _ On polarized manifolds of $\Delta$-genus two;part I, J. Math. Soc. Japan 36 (1984), $709-730$.

[F6] - On polarized manifolds whose adjoint bundles are not semipositive, Algebraic Geometry Sendai 1985, Adv. Stud. in Pure Math., vol. 10, Kinokuniya, 1987, pp. 167178.

[F7] Classification of polarized manifolds of sectional genus two, Algebraic Geometry and Commutative Algebra (Hijikata et al., eds.), Kinokuniya, 1987, pp. 73-98.

[F8] _ Ample vector bundles of small $c_{1}$-sectional genera, J. Math. Kyoto Univ. 29 (1989), 1-16.

[F9] , Polarized manifolds of degree three and $\Delta$-genus two, J. Math. Soc. Japan 41 (1989), 311-331.

[H1] R. Hartshorne, Ample vector bundles on curves, Nagoya Math. J. 43 (1971), 73-89.

[H2] Algebraic Geometry, Graduate Texts in Math., vol. 52, Springer, 1977.

[I] P. Ionescu, Embedded projective varieties of small invariants, Algebraic Geometry Bucharest 
[KMM] Y. Kawamata,K. Matsuda and K. Matsuki, Introduction to the Minimal Model Program, Algebraic Geometry Sendai 1985, Adv. Stud. in Pure Math., vol. 10, Kinokuniya, 1987, pp. 283-360.

[Ma] H. Maeda, On polarized surfaces of sectional genus three, Sci. Pap. Col. of Arts \& Sci. Univ. of Tokyo 37 (1987), 103-112.

[Mi] Y. Miyaoka, The Chern classes and Kodaira dimension of a minimal variety, Algebraic Geometry Sendai 1985, Adv. Stud. in Pure Math., vol. 10, Kinokuniya, 1987, pp. 449-476.

[OSS] C. Okonek,M. Schneider and H. Spindler, Vector Bundles on Complex Projective Spaces, Progress in Math., vol. 3, Birkhäuser, 1980.

[SW] M. Szurek and J. A. Wiśniewski, Fano bundles of rank 2 on surfaces, Compositio Math. 76 (1990), 295-305.

[V] A. Van de Ven, On uniform vector bundles, Math. Ann. 195 (1972), 245-248.

OH-OKAyAma,Meguro,TOKYo, 152, JAPAN

E-mail address: ishihara@math.titech.ac.jp 